hep-ph/0703092

HRI-P-07-03-001

OUTP 0703P

\title{
Turbulent Supernova Shock Waves and the Sterile Neutrino Signature in Megaton Water Detectors
}

\author{
Sandhya Choubey ${ }^{1 *}$, N. P. Harries ${ }^{2 \dagger}$, G.G. Ross ${ }^{2 \ddagger}$ \\ ${ }^{1}$ Harish-Chandra Research Institute, \\ Chhatnag Road, Jhunsi, Allahabad 211 019, INDIA \\ ${ }^{2}$ The Rudolf Peierls Centre for Theoretical Physics, \\ University of Oxford, 1 Keble Road, Oxford, OX1 3NP, UK
}

\begin{abstract}
The signatures of sterile neutrinos in the supernova neutrino signal in megaton water Cerenkov detectors are studied. Time dependent modulation of the neutrino signal emerging from the sharp changes in the oscillation probability due to shock waves is shown to be a smoking gun for the existence of sterile neutrinos. These modulations and indeed the entire neutrino oscillation signal is found to be different for the case with just three active neutrinos and the cases where there are additional sterile species mixed with the active neutrinos. The effect of turbulence is taken into account and it is found that the effect of the shock waves, while modifed, remain significant and measurable. Supernova neutrino signals in water detectors can therefore give unambiguous proof for the existence of sterile neutrinos, the sensitivity extending beyond that for terrestial neutrino experiments. In addition the time dependent modulations in the signal due to shock waves can be used to trace the evolution of the shock wave inside the supernova.
\end{abstract}

\footnotetext{
*email: sandhya@mri.ernet.in

$\dagger$ email: n.harries1@physics.ox.ac.uk

‡email: g.ross1@physics.ox.ac.uk
} 


\section{Introduction}

The detection of neutrinos from SN1987A in the Large Megallenic Cloud remains a major landmark in neutrino physics and astrophysics. The 11 events detected in Kamiokande [1] and 8 events in IMB [2] stimulated a plethora of research papers exploring both type-II supernova dynamics and neutrino properties [3]. A supernova explosion within our own galaxy will generate tens of thousands of events in the currently running and proposed neutrino detectors and hence is expected to significantly improve our understanding of the type-II supernova explosion mechanism on one hand and neutrino physics on the other.

Our knowledge on the pattern of neutrino mass and mixing has seen tremendous improvement from the results of a series of outstanding experiments with solar [4, reactor [5, 6], atmospheric [7] and accelerator [8, 9] neutrinos. The existence of neutrino flavor mixing and oscillations have been established beyond doubt. The $3 \sigma$ range for the mixing parameters governing solar neutrino oscillations is $11 m_{21}^{2}=(7.2-9.2) \times 10^{-5}$ $\mathrm{eV}^{2}$ and $\sin ^{2} \theta_{12}=0.25-0.39$ [10, while the atmospheric neutrino oscillation parameters are restricted to be within the range $\Delta m_{31}^{2}=2.0-3.2 \times 10^{-3}$ and $0.34<\sin ^{2} \theta_{23}<0.68$ with $\sin ^{2} 2 \theta_{23}<0.9$ [10. However, the last mixing angle $\theta_{13}$ remains unmeasured, although we do know that it must be small. The current $3 \sigma$ upper bound on the allowed values for this parameter is $\sin ^{2} \theta_{13}<0.044$ [10. If $\theta_{13}$ is close to the upper bound it opens up the possibility of observing CP violation in the lepton sector - the CP phase $\delta_{C P}$ being the second missing link in our measurement of the neutrino mass matrix. Also still unknown is $\operatorname{sgn}\left(\Delta m_{31}^{2}\right)$, which determines the ordering of the neutrino mass spectrum, aka the neutrino mass hierarchy. The case $\Delta m_{31}^{2}>0$ corresponds to the normal mass hierarchy (N3) while $\Delta m_{31}^{2}<0$ corresponds to the inverted mass hierarchy (I3) 2 A number of suggestions have been put forward to determine these three unmeasured parameters. These include the measurement of antineutrinos from reactors with detector set-up aiming to achieve sub-percent level in systematic uncertainties [1], using intense conventional $\nu_{\mu}$ beams produced by decay of accelerated pions [12, 13, using $\nu_{e}$ beams produced by decay of accelerated radioactive ions stored in rings ("Beta-Beams") 14 and using $\nu_{e}$ and $\nu_{\mu}$ beams produced by decay of accelerated muons stored in rings ("Neutrino Factory") [15]. Most of these proposed experiments are expected to be very expensive as well as technologically challenging.

In principle, information on two of the three parameters mentioned above can be obtained by observing the neutrinos released from a galactic supernova. Detailed studies on the potential of using supernova neutrinos to unravel the mass hierarchy and $\theta_{13}$ have been performed in the context of water Cerenkov detectors in [16] and (large) scintillator detectors in [17]. It was shown that the neutrino telescope, IceCube, even though designed to observe ultra high energy neutrinos, could be used very effectively to detect low energy supernova neutrinos [18, 19]. Most studies on determining of $\operatorname{sgn}\left(\Delta m_{31}^{2}\right)$ and $\theta_{13}$ with supernova neutrinos depend on the hierarchy between the average energies of $\nu_{e}, \bar{\nu}_{e}$ and $\nu_{x}$, where $\nu_{x}$ stands for $\nu_{\mu}, \bar{\nu}_{\mu}, \nu_{\tau}$, or $\bar{\nu}_{\tau}$. However the most recent supernova models which take into account the full range of the significant neutrino transport processes, predict very little difference between the average energies of $\bar{\nu}_{e}$ and $\nu_{x}$ 20. At the same time they also seem to be inconsistent with equi-partition of energy between the different neutrino species, an assumption of all earlier papers on supernova neutrinos 21]. In a nutshell, model uncertainties in the supernova parameters could washout the oscillation effects and render this method of hierarchy and $\theta_{13}$ determination useless.

Less model dependent signatures of $\operatorname{sgn}\left(\Delta m_{31}^{2}\right)$ and $\theta_{13}$ can be seen through the "shock effects" in the supernova neutrino signal in large detectors [19, 22, 23, 24, 25, 26]. The steep density profile at the shock front results in a drastic change in the oscillation probability and hence can yield information on the neutrino properties $\left(\operatorname{sgn}\left(\Delta m_{31}^{2}\right)\right.$ and $\left.\theta_{13}\right)$ despite the uncertainties associated with the supernova dynamics and neutrino transport. However it is also expected that the shock, as it moves outward, will leave behind a turbulence in the density profile of the supernova matter. Early studies indicated that this could severely obscure the oscillation signals due to the shock wave [27. Here we reexamine this in detail following a recent analysis of the the effect of turbulence in 28 . In this the nature of the shock plays an important role. In addition to the initial forward shock it is expected from supernova simulations that a reverse shock is formed 25. We find that the effects of the reverse shock are wiped out by turbulence but that the effects of the forward shock, while changed, are still significant and leave a clear signal of the resonant oscillation.

Another important question in neutrino physics is how many (if any) sterile neutrino species there are. The

\footnotetext{
${ }^{1}$ We adopt the convention in which $m_{i j}^{2}=m_{i}^{2}-m_{j}^{2}$.

${ }^{2}$ Note that the same notation is used even when the neutrino mass spectrum is quasi-degenerate since the $\mathrm{NH}$ and IH terms here refer only to the $\operatorname{sgn}\left(\Delta m_{31}^{2}\right)$.
} 
first, and so far the only, experimental evidence which requires the presence of sterile neutrinos comes from the LSND experiment [29. While the so-called $2+2$ and $3+1$ scenarios involving only 1 sterile neutrino [30] are now comprehensively disfavored by the global neutrino data, the $3+2$ scheme with 2 sterile neutrinos mildly mixed with the 3 active neutrinos has a more acceptable fit to all data including LSND 31] although the value of the associated LSND mixing angle is still problematic 32. The LSND result will be checked by the on-going MiniBOONE experiment [33] which is expected to give the final verdict at least on the oscillation interpretation of the LSND data. However, even though MiniBOONE should refute the LSND result, it will still leave ample room for the existence of extra sterile neutrinos with mixing angles below its sensitivity limit. We show that measurement of supernovae oscillation effects provide a much more sensitive probe for sterile neutrinos and analyze in detail the signal that results assuming the $3+2$ LSND scenario.

In [19] we analyzed the signature in the IceCube detector of neutrino oscillation occuring within a galactic supernova, taking shock effects into account and assuming that the $3+2$ mixing scheme was true. We considered all possible neutrino mass spectrum with 3 active and 2 sterile neutrinos and showed that IceCube could distinguish between these different mass spectra. We also showed how well IceCube could distinguish the case where there were only 3 active neutrinos from the ones where there are 2 additional sterile neutrinos. In this paper we repeat this analysis for the case in megaton water Cerenkov detectors, including the effect of turbulence. Water Čerenkov detectors have a good energy resolution and allow for the reconstruction of the energy of the incoming neutrinos. Thus, for such detectors, we will have information on both the time as well as the energy of the arriving supernova neutrinos. We calculate the number of supernova $\bar{\nu}_{e}$ events in a generic megaton water Cerenkov detector. We calculate this expected signal first for the case of three active neutrinos and then for the case with two extra sterile species. We show that the presence of sterile neutrinos changes the time evolution of the average energy as well as the flux of the supernova neutrinos. Therefore, this can be used to indicate the presence of sterile neutrino and also to distinguish between the different possible mass spectrum scenarios. The effect of the shock is shown to be significant on the resultant signal. We discuss the issue of turbulence and take into account the turbulence due to the passage of shock.

The paper is organized as follows. We begin in Section 2 with a brief review of neutrino oscillations inside supernova. We discuss the effect of the shock wave on the oscillation probability, with and without the turbulence caused by the passage of shock. The impact on the neutrino oscillation probability by the turbulence in the matter density behind the shock is clearly outlined. In Section 3 we discuss the neutrino spectrum produced inside the supernova and their mode of detection in water Cerenkov experiments. In section 4 we present our results and discuss the implications in Section 5. We end with our conclusions in Section 6.

\section{Neutrino Oscillations Inside the Supernova}

When neutrinos propagate in matter, they pick up an extra potential energy induced by their charged current and neutral current interactions with the ambient matter [34, 35, 36]. Since normal matter only contains electrons, only $\nu_{e}$ and $\bar{\nu}_{e}$ undergo both charged as well as neutral current interactions, while the other 4 active neutrino species have only neutral current interactions. The evolution equation of (anti)neutrinos inside the supernova can be written in the flavor basis as

$$
\mathcal{H}=\frac{1}{2 E}\left(U \mathcal{M}^{2} U^{\dagger}+\mathcal{A}\right),
$$

where $U$ is a unitary matrix and is defined by $\left|\nu_{i}\right\rangle=\sum_{\alpha} U_{i \alpha}\left|\nu_{\alpha}\right\rangle, \nu_{i}$ and $\nu_{\alpha}$ being the mass and flavor eigenstates respectively. For the case where we consider two extra sterile neutrinos, $i=1-5$ and $\alpha=\mathrm{e}, \mu, \tau, s_{1}$ or $s_{2}$, where $s_{1}$ and $s_{2}$ are the two sterile neutrinos. The matrices $\mathcal{M}^{2}$ and $\mathcal{A}$ are given respectively by

$$
\begin{gathered}
\mathcal{M}^{2}=\operatorname{Diag}\left(m_{1}^{2}, m_{2}^{2}, m_{3}^{2}, m_{4}^{2}, m_{5}^{2}\right), \\
\mathcal{A}=\operatorname{Diag}\left(A_{1}, 0,0, A_{2}, A_{2}\right), \\
A_{1}=A_{C C}= \pm \sqrt{2} G_{F} \rho N_{A} Y_{e} \times 2 E, \\
A_{2}=-A_{N C}= \pm \sqrt{2} G_{F} \rho N_{A}\left(1-Y_{e}\right) \times E .
\end{gathered}
$$


The quantities $A_{C C}$ and $A_{N C}$ are the matter induced charged current and neutral current potentials respectively and depend on the Fermi constant $G_{F}$, matter density $\rho$, Avagadro number $N_{A}$, electron fraction $Y_{e}$ and energy of the neutrino E. The "+" ("-") sign in Eqs. (4) and (5) corresponds to neutrinos (antineutrinos).

The corresponding expressions for the three neutrino framework follows simply by dropping the extra 2 states due to the sterile components. Note that we have recast the matter induced mass matrix such that the neutral current part $A_{N C}$, which appears for all the three active flavors, is filtered out from the first three diagonal terms and hence it appears as a negative matter potential for the sterile states which do not have any weak interactions. Therefore, for the three active neutrino set-up the mass matrix is

$$
\begin{gathered}
\mathcal{M}^{2}=\operatorname{Diag}\left(m_{1}^{2}, m_{2}^{2}, m_{3}^{2}\right), \\
\mathcal{A}=\operatorname{Diag}\left(A_{1}, 0,0\right) .
\end{gathered}
$$

The mixing matrix $U$ is given in terms of mixing angles and CP-violating phases. If CP conservation is assumed the mixing matrix takes the form

$$
U=\prod_{B>A}^{n} \prod_{A=1}^{n-1} R^{A B\left(\theta_{A B}\right)},
$$

where $n=3$ for only three active neutrinos and $n=5$ when two additional sterile neutrinos are present and $R^{A B}$ is an $n \times n$ rotation matrix about the $\mathrm{AB}$ plane.

Assuming that all phases get averaged out, the survival probability $\bar{P}_{e e}$ of $\bar{\nu}_{e}$ after they have propagated through the supernova matter can be written a 3

$$
\bar{P}_{e e}=\sum_{i, j}\left|U_{e j}\right|^{2}\left|U_{e i}^{m}\right|^{2} P_{i j}
$$

where, $P_{i j}=\left|\left\langle\bar{\nu}_{j} \mid \bar{\nu}_{i}^{m}\right\rangle\right|^{2}$ and $U_{e i}$ and $U_{e i}^{m}$ are the elements of the mixing matrix in vacuum and inside matter respectively and $\left|\left\langle\bar{\nu}_{j} \mid \bar{\nu}_{i}^{m}\right\rangle\right|^{2}$ is the effective "level crossing" probability that an antineutrino state created as $\left|\bar{\nu}_{i}^{m}\right\rangle$ inside the supernova core emerges as the state $\left|\bar{\nu}_{i}\right\rangle$ in vacuum. Largest flavor conversions occur at the resonance densities. For two flavor oscillations, the resonance condition for antineutrinos involving only either the $\bar{\nu}_{e}$ or the sterile states is given by 35 .

$$
\left|A_{k}\right|=(-1)^{k} \Delta m_{j i}^{2} \cos 2 \theta_{i j}
$$

where $k$ could be either 1 or $2, \Delta m_{j i}^{2} \equiv m_{j}^{2}-m_{i}^{2}$ is the mass squared difference and $\theta_{i j}$ is the mixing angle between the two states in vacuum. Note in Eq. (10), we included the sign factor $(-1)^{k}$ in order to take into account the fact that $A_{C C}<0$ and $A_{N C}>0$ for the antineutrinos. Since $A_{C C}<0\left(A_{N C}>0\right)$, the condition of resonance is satisfied only when the relevant $\Delta m_{j i}^{2}<0\left(\Delta m_{j i}^{2}>0\right)$. For the resonance between the $\bar{\nu}_{e}$ and sterile states one must satisfy the condition

$$
A_{C C}-A_{N C}=\Delta m_{j i}^{2} \cos 2 \theta_{i j}
$$

which is satisfied for antineutrinos when $\Delta m_{j i}^{2}<0$ since $A_{C C}>A_{N C}$.

The probability of level crossing from one mass eigenstate to another is predominantly non-zero only at the resonance. In the approximation that the individual two flavor resonances are far apart, the effective level crossing probability can be written in terms of the individual two flavor "flip probability" $P_{i j}$. The "flip probability" between the two mass eigenstates used in this paper is given by [38]

$$
\begin{gathered}
P_{i j}=\frac{\exp \left(-\gamma \sin ^{2} \theta_{i j}\right)-\exp (-\gamma)}{1-\exp (-\gamma)}, \\
\gamma=\pi \frac{\Delta m_{j i}^{2}}{E}\left|\frac{d \ln A}{d r}\right|_{r=r_{m v a}}^{-1},
\end{gathered}
$$

\footnotetext{
${ }^{3}$ Since we are interested in the detection of supernova neutrino in water Čerenkov detectors where the largest number of events are expected through the capture of $\bar{\nu}_{e}$ by protons, we will mostly refer to the survival probability of $\bar{\nu}_{e}\left(\bar{P}_{e e}\right)$ in this section. However, similar expressions are valid even for the $\nu_{e}$ survival probability.
} 
where $A$ is $A_{C C}, A_{N C}$ or $A_{C C}-A_{N C}$ depending on the states involved in the resonance, $r_{m v a}$ is the position of the maximum violation of adiabaticity ( $\mathrm{mva}$ ) [37] and is defined as

$$
A\left(r_{m v a}\right)=\Delta m_{j i}^{2} .
$$

One can see from the above equations that $P_{i j}$ and hence the transition probability depends crucially both on the mixing angle as well as on the density gradient. If for given $\Delta m_{j i}^{2}$ and $\theta_{i j}$, the density gradient is small enough so that $\gamma \gg 1$ and $\gamma \sin ^{2} \theta \gg 1, P_{i j} \simeq 0$ and the transition is called adiabatic. On the other hand, if the density gradient is very large or $\theta_{i j}$ is very small, then $P_{i j} \simeq \cos ^{2} \theta_{i j}$. In this case we have an extreme non-adiabatic transition. For all intermediate values of the density gradient and $\theta_{i j}, P_{i j}$ ranges between [0-1].

\subsection{Neutrino Oscillations with Static Density Profile}

Three active neutrinos only: As discussed above, in the three neutrino framework we have two possibilities for the neutrino mass spectrum, the normal $\left(\Delta m_{31}^{2}>0\right)$ and inverted $\left(\Delta m_{31}^{2}<0\right)$ hierarchy. We will call them N3 and I3 cases respectively. Also, since here we have 2 independent $\Delta m_{j i}^{2}$, we can have 2 resonances and hence the final level crossing probability will be given in terms of the 2 individual flip probabilities. However, it is now known at more than $6 \sigma$ C.L. from the solar neutrino data that $\Delta m_{21}^{2}>0$, and hence the $\Delta m_{21}^{2}$ driven resonance condition given by Eq. (10) is satisfied only for the neutrino channel. But the sign of $\Delta m_{31}^{2}$ is still unknown. Hence the $\Delta m_{31}^{2}$ driven resonance can appear in either the neutrino or antineutrino sector depending on whether the mass hierarchy turns out to be normal or inverted respectively. Therefore, for $\Delta m_{31}^{2}<0$, the $\bar{\nu}_{e}$ survival probability (neglecting earth matter effects) is given by

$$
\bar{P}_{e e}=P_{13}\left|U_{e 1}\right|^{2}+\left(1-P_{13}\right)\left|U_{e 3}\right|^{2}
$$

where $P_{13}$ is the flip probability between the 1 and 3 states and can be calculated using Eq. (12) with $\Delta m_{j i}^{2} \equiv$ $\Delta m_{31}^{2}$ and $\theta_{i j} \equiv \theta_{13}$. For $\Delta m_{31}^{2}>0$ the resonance does not occur in the antineutrino channel and therefore

$$
\bar{P}_{e e}=\left|U_{e 1}\right|^{2}
$$

Three active plus two sterile neutrinos: For the three active and two sterile neutrino framework we have 4 independent mass squared differences and hence can have as many as six possibilities for the mass spectrum which we call [19]:

$$
\begin{array}{rll}
\text { N2+ N3 }: & \Delta m_{31}^{2}>0 & , \Delta m_{41}^{2}>0 \text { and } \Delta m_{51}^{2}>0, \\
\text { N2 + I3: } & \Delta m_{31}^{2}<0 & , \Delta m_{41}^{2}>0 \text { and } \Delta m_{51}^{2}>0, \\
\text { H2 + N3 }: & \Delta m_{31}^{2}>0 & , \Delta m_{41}^{2}>0 \text { and } \Delta m_{51}^{2}<0, \\
\text { H2 + I3 }: & \Delta m_{31}^{2}<0 & , \Delta m_{41}^{2}>0 \text { and } \Delta m_{51}^{2}<0, \\
\text { I2 + N3 }: & \Delta m_{31}^{2}>0 & , \Delta m_{41}^{2}<0 \text { and } \Delta m_{51}^{2}<0, \\
\text { I2 + I3 }: & \Delta m_{31}^{2}<0 & , \Delta m_{41}^{2}<0 \text { and } \Delta m_{51}^{2}<0,
\end{array}
$$

with $\Delta m_{21}^{2}>0$ always. Since we have 4 different independent $\Delta m_{j i}^{2}$ and both active and sterile components, we can have many more resonances in this case. In fact, we expect to have resonances between the 1-2, 1-3, 1-4 and 1-5 states due to the charged current term and between 2-4, 2-5, 3-4 and 3-5 states due to the neutral current term. Again since $\Delta m_{21}^{2}>0$, the 1-2 resonance happens only for neutrinos, while the other resonances can happen in either the neutrino or antineutrino channel depending on whether the corresponding mass squared difference is positive or negative. Another difference between the only active and active plus sterile cases is that while for only active neutrino cases it was enough to compute just the survival probability $\bar{P}_{e e}$, with sterile neutrinos in the fray, the total neutrino flux produced inside the supernova does not remain constant as the active flavors also disappear into sterile species. Thus we will need to calculate the probabilities $\bar{P}_{e e}$ as well as $\bar{P}_{x e}$. The oscillation probability in general is given by

$$
\bar{P}_{\alpha \beta}=\sum_{i} \bar{P}_{\alpha i}^{m} \bar{P}_{i \beta}^{\oplus},
$$




\begin{tabular}{|c|c|c|c|}
\hline Hierarchy & $\mathrm{i}$ & $\bar{P}_{e i}^{m}$ & $\bar{P}_{x i}^{m}$ \\
\hline $\mathrm{N} 2+\mathrm{N} 3$ & $\begin{array}{l}1 \\
2 \\
3 \\
4 \\
5\end{array}$ & $\begin{array}{l}1 \\
0 \\
0 \\
0 \\
0\end{array}$ & $\begin{array}{c}0 \\
P_{25} P_{24} \\
P_{25}\left(1-P_{24}\right)\left(1-P_{34}\right)+\left(1-P_{25}\right)\left(1-P_{35}\right) P_{34}+P_{35} P_{34} \\
P_{25}\left(1-P_{24}\right) P_{34}+\left(1-P_{25}\right)\left(1-P_{35}\right)\left(1-P_{34}\right)+P_{35}\left(1-P_{34}\right) \\
\left(1-P_{35}\right)+\left(1-P_{25}\right) P_{35}\end{array}$ \\
\hline $\mathrm{N} 2+\mathrm{I} 3$ & $\begin{array}{l}1 \\
2 \\
3 \\
4 \\
5\end{array}$ & $\begin{array}{c}P_{13} \\
0 \\
1-P_{13} \\
0 \\
0\end{array}$ & $\begin{array}{c}P_{35} P_{34}\left(1-P_{13}\right) \\
P_{35}\left(1-P_{34}\right)\left(1-P_{24}\right)+\left(1-P_{35}\right)\left(1-P_{25}\right) P_{24}+P_{25} P_{24} \\
P_{35} P_{34} P_{13} \\
P_{35}\left(1-P_{34}\right) P_{24}+\left(1-P_{35}\right)\left(1-P_{25}\right)\left(1-P_{24}\right)+P_{25}\left(1-P_{24}\right) \\
\left(1-P_{25}\right)+\left(1-P_{35}\right) P_{25}\end{array}$ \\
\hline $\mathrm{H} 2+\mathrm{N} 3$ & $\begin{array}{l}1 \\
2 \\
3 \\
4 \\
5\end{array}$ & $\begin{array}{c}P_{14} \\
0 \\
0 \\
1-P_{14} \\
0\end{array}$ & $\begin{array}{c}0 \\
P_{25} \\
P_{35}+\left(1-P_{25}\right)\left(1-P_{35}\right) \\
0 \\
\left(1-P_{35}\right)+\left(1-P_{25}\right) P_{35}\end{array}$ \\
\hline $\begin{array}{l}\mathrm{H} 2+\mathrm{I} 3 \\
\end{array}$ & $\begin{array}{l}1 \\
2 \\
3 \\
4 \\
5\end{array}$ & $\begin{array}{c}P_{14} P_{13} \\
0 \\
\left(1-P_{13}\right) P_{14} \\
\left(1-P_{14}\right) \\
0\end{array}$ & $\begin{array}{c}P_{35}\left(1-P_{13}\right) \\
\left(1-P_{35}\right)\left(1-P_{25}\right)+P_{25} \\
P_{35} P_{13} \\
0 \\
\left(1-P_{25}\right)+\left(1-P_{35}\right) P_{25}\end{array}$ \\
\hline $\mathrm{I} 2+\mathrm{N} 3$ & $\begin{array}{l}1 \\
2 \\
3 \\
4 \\
5\end{array}$ & $\begin{array}{c}P_{15} P_{14} \\
0 \\
0 \\
P_{15}\left(1-P_{14}\right) \\
1-P_{15}\end{array}$ & $\begin{array}{l}0 \\
1 \\
1 \\
0 \\
0\end{array}$ \\
\hline $\mathrm{I} 2+\mathrm{I} 3$ & $\begin{array}{l}1 \\
2 \\
3 \\
4 \\
5\end{array}$ & $\begin{array}{c}P_{15} P_{14} P_{13} \\
0 \\
P_{15} P_{14}\left(1-P_{13}\right) \\
P_{15}\left(1-P_{14}\right) \\
1-P_{15}\end{array}$ & $\begin{array}{c}1-P_{13} \\
1 \\
P_{13} \\
0 \\
0\end{array}$ \\
\hline
\end{tabular}

Table 1: The probabilities $\bar{P}_{e i}^{m}$ and $\bar{P}_{x i}^{m}$ for three active neutrinos plus two sterile neutrinos, where $\bar{P}_{\alpha i}^{m}$ is given by Eq. (25) and $P_{i j}$ is the flip probability at the resonance between the $\nu_{i}$ and $\nu_{j}$ mass eigenstates. 
where $P_{\alpha i}^{m}$ and $P_{i \beta}^{\oplus}$ are respectively the $\bar{\nu}_{\alpha} \rightarrow \bar{\nu}_{i}^{m}$ transition probability in the supernova and $\bar{\nu}_{i}^{\oplus} \rightarrow \bar{\nu}_{\beta}$ transition probability in the earth. In the absence of earth matter effects

$$
\bar{P}_{i e}^{\oplus}=\left|U_{e i}\right|^{2}
$$

and

$$
\bar{P}_{\alpha i}^{m}=\sum_{j}\left|U_{\alpha j}^{m}\right|^{2} P_{i j}
$$

where $P_{i j}$ is the flip probability between the $i$ and $j$ mass eigenstates, given by Eq. (12). We explicitly give the probabilities $\bar{P}_{e i}^{m}$ and $\bar{P}_{x i}^{m}$ for all the possible mass spectra in Table 1 For further details and the level crossing diagrams for each of these mass spectra, we refer the reader to [19.

\subsection{Effect of the Shock Wave on $\bar{P}_{e e}$}

It is believed that when the core of a collapsing supernova reaches nuclear density the collapse rebounds forming a strong outward shock. The shock stalls and is eventually regenerated via neutrino heating. Numerical simulations show that as well as a forward shock a reverse shock forms and the region behind the shock wave is highly turbulent [25. The detailed density profiles from numerical simulations are not available to us, therefore we consider the simplified profile for the shock wave used in [23, 26]. A schematic diagram showing the density change at the shock front for forward shock and for forward and reverse shock is shown by the dashed black lines in Figs. 1(a) and 2(a).

As can be seen from these figures, the effect of the supernova shock wave is to generate very sharp changes in the density gradient, which is expected to change the flip probability. Since, as mentioned above, the flip probability is calculated at the position of resonance, the effect of the shock will be to modify the flip probability and hence $\bar{P}_{e e}$ when the shock crosses the resonance density for a certain $\Delta m_{j i}^{2}$. Also, we see from the figures that the shock generates fluctuations in the density gradient. This results in the same $\Delta m_{j i}^{2}$ producing multiple resonances which are relatively close together. If we assume that the phase effects can be neglected even in this case [39, then the individual resonances can be considered as independent two generation resonances and the net flip probability $P_{H}$ can be expressed in terms of the multiple flip probabilities $P_{i}$ as [40, 23]

$$
\left(\begin{array}{cc}
1-P_{H} & P_{H} \\
P_{H} & 1-P_{H}
\end{array}\right)=\prod_{i=1, n}\left(\begin{array}{cc}
1-P_{i} & P_{i} \\
P_{i} & 1-P_{i}
\end{array}\right)
$$

where $n$ is the number of resonances occurring for the same $\Delta m_{j i}^{2}$ due to the shock effect. In particular, if the mixing angles are sufficiently large then in the region of the supernova where the density can be approximated as almost static, the flip probability between the mass eigenstates is approximately zero (the resonance is adiabatic). As the shock wave passes through the resonant density, the flip probability increases to approximately one (the resonance becomes strongly non-adiabatic). As the shock passes over, then in the approximation that there is no turbulence (which will be considered in the next subsection), the flip probability goes back to zero. The resultant flip probability has a time dependence shown in Figs. 1(b) and 2(b).

Since the resonance densities are different for the different $\Delta m_{j i}^{2}$ involved, the shock wave will cross them at different times. Therefore, one can follow the evolution of the shock wave by following the time profile of the supernova signal [19]. Also, since the resonance density is determined by the energy of the neutrino, different energy neutrinos have their resonance position as different points and thus get affected by the shock at different times. Thus the shock imprints its signature on both the energy spectrum as well as the time profile of the neutrinos arriving on earth. The effect of the shock wave is discussed in 22, 23, for the forward shock and in [25, 26] when there is a reverse shock as well. All these papers used large/megaton water detectors for the supernova neutrino signal on earth. The possibility of using shock waves to discern the existence of eV mass sterile neutrinos in the supernova signal in IceCube was discussed in [19].

\subsection{Turbulence}

Numerical simulations have shown that a highly turbulent region forms behind the shock wave [41. The fluctuations in these turbulence cause flips between the mass eigenstates [27, 28. The length scale of the fluctuations for which the flips are dominant are at the length scale 

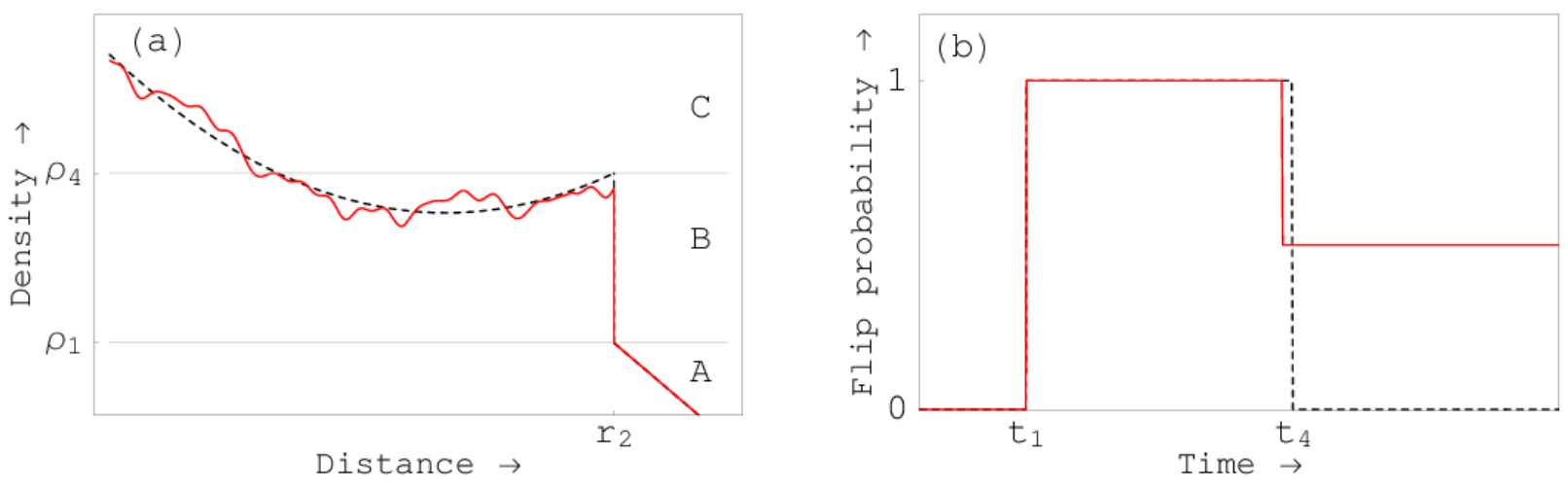

Figure 1: (a) The density profile of the forward shock as a function of the distance from the core of the supernova. (b) The resulting flip probability as a function of time. The dashed black line is for the case of no turbulence and the solid red line includes the effect of turbulence.
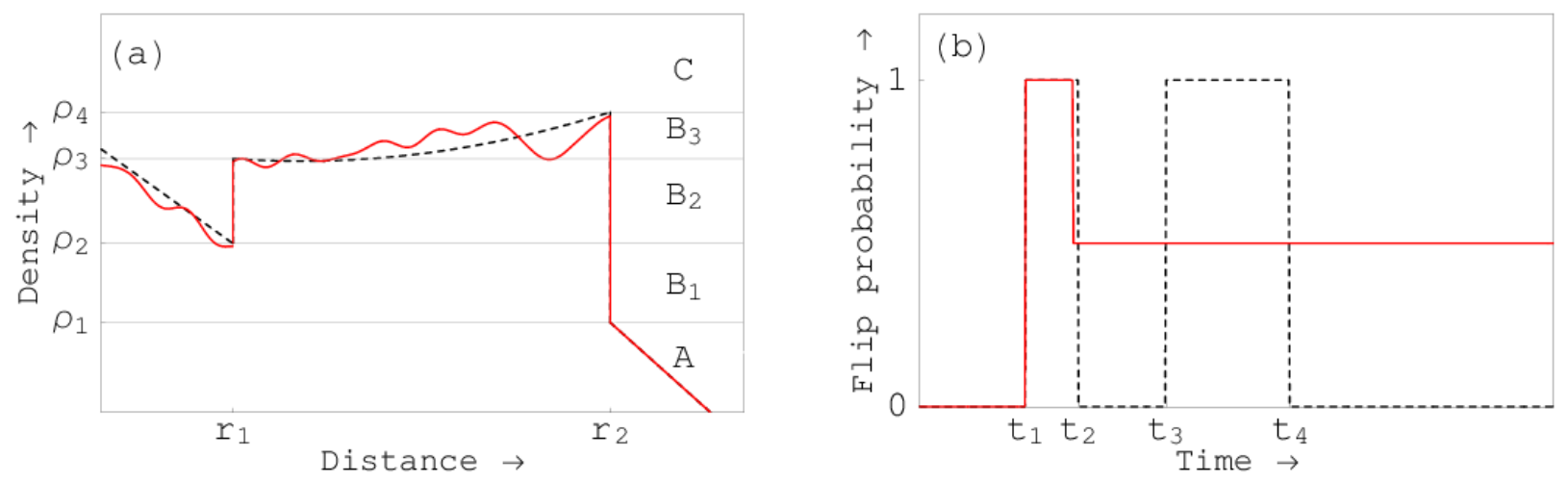

Figure 2: (a) The density profile of the forward and reverse shock as a function of the distance from the core of the supernova. (b) The resulting flip probability as a function of time. The dashed black line is for the case of no turbulence and the solid red line includes the effect of turbulence.

$$
L_{\text {fluct }}=\frac{4 \pi E}{\Delta m^{2} \sin 2 \theta}
$$

For the atmospheric resonance $L_{\text {fluct }}=47 \mathrm{~km} \frac{E}{15 \mathrm{MeV}} \frac{0.3}{\sin 2 \theta_{13}}$, which is smaller than the resolution of the numerical simulations and therefore a physical model of the fluctuations is required. Analytical solutions for a density fluctuating medium have only been found for the un-physical delta-correlated noise

$$
\langle\delta n(x) \delta n(y)\rangle=2 L_{0} \delta n^{2} \delta(x-y)
$$

where the fluctuations are fully correlated for length scales less than $L_{0}$ and $\delta n^{2}$ is the average squared fluctuation. Simulations using these analytical solutions show that the system becomes depolarized for large fluctuations, the flip probability $P_{\text {turb }} \sim 0.5$.

A more physically reasonable model of turblence is provided by the Kolmogorov spectrum in which the large scale density fluctuations cascade into density fluctuations on a small scale. These density fluctuations exhibit a power-law spectrum 


$$
\int d x\langle\delta n(0) \delta n(x)\rangle e^{-i k x}=C_{0} k^{\alpha}
$$

where $n(x)$ is the density of the supernova at the position $x, k=2 \pi / \lambda$ where $\lambda$ is the wavelength of the fluctuation, $C_{0}$ is a constant and $\alpha=-5 / 3$. The density fluctuations on a large scale (the size of the shock wave) can be taken from numerical simulations and used to determine the value of $C_{0}$. Then this can be used to estimate the size of the fluctuations at the scale at which dominate the oscillations. Numerical solutions for the case of Kolmogorov turbulence also show that the system becomes depolarized for large turbulence [28. Currently no exact solutions exist for Kolmogorov turbulence but the flip probability can be calculated in the perturbative limit giving

$$
P_{\text {turb }} \simeq\left\{\begin{array}{ccc}
\frac{0.84 G_{F} C_{0}}{\sqrt{2}\left|n_{0}^{\prime}\right|}\left(\frac{\Delta m^{2} \sin 2 \theta}{2 E}\right)^{-2 / 3} & \text { for } & \frac{\pi\left(\Delta m^{2} \sin 2 \theta\right)^{2}}{4 E\left|A^{\prime}\right|} \gg 1 \\
1 & \text { for } & \frac{\pi\left(\Delta m^{2} \sin 2 \theta\right)^{2}}{4 E\left|A^{\prime}\right|} \ll 1
\end{array}\right.
$$

This is in the perturbative limit but a depolarization criterion can be defined by $P_{t u r b} \sim 0.5$. Fluctuations which satisfy this condition are expected to depolarize the neutrino flavours which are maximally mixed at the resonance. The current simulations show that this criteria is satisfied by a large margin for both the atmospheric and sterile resonances. In these cases the resonant neutrinos are expected to be depolarized in the turbulent region behind the shock wave.

The flip probability of the density profile including the effect of turbulence is shown in Figs. 11 and 2. Note that for the forward shock the initial rise is unchanged by turbulence but the fall is reduced by the turbulent depolarisation. This just reflects the fact that the turbulence follows the shock wave. The effect on the reverse shock is dramatic, the depolarisation wiping out the reverse shock structure. Note too that the depolarisation following the shock front can lead to interesting observable effects in active neutrino oscillation for the case there are additional sterile neutrinos. This is because the active-sterile oscillation will destroy the near degeneracy between $\overline{\nu_{e}}$ and $\nu_{x}$. As a result the subsequent atmospheric neutrino oscillation signal, which vanishes if the active neutrino luminosities and spectra are equal, can be significantly enhanced. We will demonstrate these effects in the subsequent sections.

\section{Observing Supernova Neutrinos in Water}

\subsection{Energy Spectra of Supernova Neutrinos}

In a type-II supernova approximately $3 \times 10^{53}$ ergs of energy is released and about $99 \%$ of this is in the form of neutrinos. The energy spectra of these neutrinos is determined by their interactions with matter. As the effect of weak magnetism in muon or tau production can be neglected, the spectra of $\nu_{\mu}, \bar{\nu}_{\mu}, \nu_{\tau}$ and $\bar{\nu}_{\tau}$ are approximately equal and are collectively denoted by $\nu_{x}$. The initial spectra of neutrino species $\nu_{\alpha}$ from a supernova is parameterized as 42 ]

$$
F^{0}(E, t)=\frac{\Phi(t)}{\langle E\rangle(t)} \frac{(\alpha(t)+1)^{\alpha(t)+1}}{\Gamma(\alpha(t)+1)}\left(\frac{E}{\langle E\rangle(t)}\right)^{\alpha(t)} \exp \left(-(\alpha(t)+1) \frac{E}{\langle E\rangle(t)}\right)
$$

where $\langle E\rangle$ and $\Phi$ are the average energy and total number flux and $\alpha$ is a dimensionless parameter which typically takes the values 2.5-5. For the results presented in this paper, we have assumed $\alpha_{\bar{e}}=3$ and $\alpha_{x}=4$.

To date only the Lawrence Livermore (LL) group 21] has published detailed results of the energy spectra of neutrinos for the duration of the supernova. In the LL simulations the luminosities of all flavours of neutrinos are approximately equal for times post bounce $\gtrsim 0.1 \mathrm{~s}$. However there is a distinct difference in the average energies. This is due to the additional charged current interactions of the $\nu_{e}$ and $\bar{\nu}_{e}$ with ambient matter. However these simulations did not include all the neutrino interactions now believed to be important 20. Ongoing work by the Garching group including all possibly significant interactions predict the neutrino fluxes and average energies labeled G1 and G2 in Table 2. This shows that the average energies between neutrino flavours become very similar and the luminosities are no longer equal. In order to compare the impact of the uncertainties on the average energies and fluxes of the neutrinos obtained in different supernova computer simulations, we will 


\begin{tabular}{|c|c|c|c|c|c|}
\hline Model & $\left\langle E_{\nu_{e}}^{0}\right\rangle$ & $\left\langle E_{\bar{\nu}_{e}}^{0}\right\rangle$ & $\left\langle E_{\nu_{x}}^{0}\right\rangle$ & $\frac{\Phi_{\nu_{e}}^{0}}{\Phi_{\nu_{x}}^{0}}$ & $\frac{\Phi_{\bar{\nu}_{e}}^{0}}{\Phi_{\nu_{x}}^{0}}$ \\
\hline LL & 12 & 15 & 24 & 2.0 & 1.6 \\
G1 & 12 & 15 & 18 & 0.8 & 0.8 \\
G2 & 12 & 15 & 15 & 0.5 & 0.5 \\
\hline
\end{tabular}

Table 2: The average energies and total fluxes characterizing the primary neutrino spectra produced inside the supernova. The numbers obtained in the Lawrence Livermore simulations are denoted as LL, while those obtained by the Garching group are denoted as G1 and G2.

present our results using supernova neutrino parameters given by both the Lawrence Livermore and Garching groups. Specifically, we consider the three cases shown in Table 2 25. For further details, we refer the reader to our earlier paper [19].

\subsection{Signal in Water Cerenkov Detectors}

Supernova neutrinos with energy in the $\mathrm{MeV}$ regime will be dominantly detected in water through the capture of $\bar{\nu}_{e}$ on protons

$$
\bar{\nu}_{e}+p \rightarrow n+e^{+}
$$

The emitted positron will be observed in the detector and its energy and time measured. Hence we should be able to get a fairly good reconstruction of the incoming $\bar{\nu}_{e}$ energy spectrum and time profile. Neglecting the recoil of the neutron the energy of the neutrino $E$ is related to the energy of the positron through the relation $E_{e}=E-1.29$, where all energies are in $\mathrm{MeV}$. The number of events expected in a water Cerenkov detector from a galactic supernova explosion is given by

$$
N=\frac{N_{T}}{4 \pi D^{2}} \int_{0}^{\infty} \int_{0}^{\infty} F(E) * \sigma(E) * \varepsilon(E) * R\left(E-1.29, E_{e}\right) d E d E_{e}
$$

where $D$ is the distance of the supernova from earth, $N_{T}$ is the number of target nucleons in 1 megaton of water, $\mathrm{E}$ is the energy of the neutrino, $E_{e}$ is the measured energy of the positron, $\mathrm{F}(\mathrm{E})$ is the flux at the detector, as defined in Eqs. (30) and (37), $\sigma(E)$ is the cross section, $\varepsilon(E)$ is the efficiency of detection and $R\left(E-1.29, E_{e}\right)$ is the energy resolution function. The efficiency is assumed to be perfect above $7 \mathrm{MeV}$ and vanishing below this energy. The energy resolution function for which we assume a Gaussian form

$$
R\left(E_{T}, E_{e}\right)=\frac{1}{\sqrt{2 \pi} \sigma_{E}} \exp \left(\frac{-\left(E_{T}-E_{e}\right)^{2}}{2 \sigma_{E}^{2}}\right)
$$

where $E_{T}$ and $E_{e}$ are respectively the true and measured energy of the positron and we take the HWHM $\sigma_{E}\left(E_{T}\right)=\sqrt{E_{0} E_{T}}$, where $E_{0}=0.22 \mathrm{MeV}$. This is the same efficiency and energy resolution as used in 25, 43. Water Cerenkov detectors usually are expected to have time resolution which is of the order of nanosecond. In what follows, we will bin our data either in time bins of $100 \mathrm{~ms}$ (at later times) or $10 \mathrm{~ms}$ (at earlier times). Since we expect the time resolution of the detector to be at least 3-4 orders of magnitude better, we do not include any time resolution function in our calculation of the number of events.

In addition to the total number of events, we also calculate the the average energy of the detected positrons through the expression

$$
\left\langle E_{e}\right\rangle=\frac{\int_{0}^{\infty} \int_{0}^{\infty} E_{A} * F(E) * \sigma(E) * \varepsilon(E) * R\left(E-1.29, E_{A}\right) d E d E_{A}}{\int_{0}^{\infty} \int_{0}^{\infty} F(E) * \sigma(E) * \varepsilon(E) * R\left(E-1.29, E_{A}\right) d E d E_{A}}
$$

\footnotetext{
${ }^{4}$ Water C̆ Cerenkov detectors have other detection channels whereby they can observe $\nu_{e}$ and $\nu_{x}$ (electron scattering and charged and neutral current interactions on ${ }^{16} \mathrm{O}$ ). However, the cross-section for these processes are much smaller and hence they are not considered here.
} 
We show both the number of events as well as the average energy as a function of time. We also show the statistical uncertainties expected in 1 megaton water Cerenkov detectors. The statistical error in the total number of events are estimated as

$$
\sigma_{N}=\sqrt{N}
$$

while that in the average energy is calculated as

$$
\sigma_{\langle E\rangle}=\sqrt{\frac{\left\langle E_{e}^{2}\right\rangle-\left\langle E_{e}\right\rangle^{2}}{N}},
$$

where $\sigma_{\langle E\rangle}$ is the error in the average energy, $\mathrm{N}$ is the number of events, $\langle E\rangle$ is the average energy and $\left\langle E^{2}\right\rangle$ is the average energy squared.

The neutrino flux in the detector is

$$
F_{\beta}=\sum_{\alpha} F_{\alpha}^{0} P_{\alpha \beta}
$$

where $P_{\alpha \beta}$ is the oscillation probability and is given in Eqs. (23)-(25) and $F_{\alpha}^{0}$ is the initial flux of $\nu_{\alpha}$ given in Eqn. (31).

\subsection{Input Supernova and Oscillation Parameters}

In what follows, we will present results for the typical values for the fluxes and average energies given in Table 2 for the LL, G1 and G2 "models". For the neutrino oscillation parameters, we assume the best-fit values $\Delta m_{21}^{2}=8 \times 10^{-5} \mathrm{eV}^{2}, \sin ^{2} \theta_{12}=0.31$ and $\Delta m_{31}^{2}=2.5 \times 10^{-3}\left[10\right.$. For $\theta_{13}$, we assume that it is large enough so that away from the shock, the $\Delta m_{31}^{2}$ driven resonant transition is fully adiabatic. Typically, this would be satisfied for $\sin ^{2} \theta_{13} \gtrsim 10^{-3}$.

The other oscillation parameters relevant for supernova neutrino oscillations in the $3+2$ scenario are constrained by the combined data from Bugey, CHOOZ, CCFR84, CDHS, KARMEN, NOMAD, and LSND [29, 44]. If we restrict the mass squared differences to lie in the sub-eV regime then the best-fit comes at $\Delta m_{41}^{2}=0.46$ $\mathrm{eV}^{2}, \Delta m_{51}^{2}=0.89 \mathrm{eV}^{2}, U_{e 4}=0.09, U_{e 5}=0.125, U_{\mu 4}=0.226$ and $U_{\mu 5}=0.16$ [31. These values of the oscillation parameters give fully adiabatic transition at the resonance in the supernova when the shock is not present.

\subsubsection{Sterile neutrino sensitivity}

In the detailed estimates presented below we use the sterile neutrino parameters consistent with an explanation of the LSND experiment. However it is important to stress that the sensitivity to sterile neutrinos is much better than is needed to probe the LSND range and that, even if MiniBoone should rule out the sterile mixing angle regime used in our estimates, supernovae neutrino signals will still be important in the search for evidence for sterile neutrinos. It is straightforward to quantify the range of sensitivity. To observe the time dependent effects in the signal due to sterile neutrinos, the adiabaticity of the sterile resonances needs to be changed by the shock wave and/or the turbulence. For mixing angles $\sin ^{2} \theta_{i j} \lesssim 4 \times 10^{-6}$ the sterile resonances would be non-adiabatic for the entire time of interest. As a result there would be no oscillations into sterile neutrinos and the signal would be equivalent to that of only 3 active neutrinos. For $\sin ^{2} \theta_{i j} \gtrsim 4 \times 10^{-4}$ the resonance would be adiabatic in the regions behind (with no turbulence) and in front of the shock wave. In this range the active-sterile resonant mixig effects are measureable. The situation is summarised in Table 3 .

For $4 \times 10^{-6}<\sin ^{2} \theta_{i j}<4 \times 10^{-4}$ there is a "transition region", in which the effects discussed in this paper could be observed but may be less prominent. To quantify this first note that within this region of parameter space the mixing angles are sufficiently large such that each resonance is adiabatic in the absence of the shock wave. As well as changing the adiabaticity of each resonance the mixing angles change the relative proportion of each flavour eigenstate in each mass eigenstate, as a result changing the $\bar{\nu}_{e}$ flux, $F_{e}$ in the detector. The approximate $F_{e}$ for small sterile mixing angles is shown in Table 4 . If the sterile mixing angles and $\theta_{13}$ are scaled as $\theta_{i j} \rightarrow k \theta_{i j}$, where $\mathrm{i}=1,2$, and $\mathrm{j}=3-5$, and $k<1, F_{e}$ is approximately unchanged except for the $\mathrm{N} 2+\mathrm{I} 3$ and $\mathrm{H} 2+\mathrm{N} 3$ mass hierarchies. If the flux is unchanged both the number of events and the average energies are 


\begin{tabular}{|c|c|}
\hline $\sin ^{2} \theta_{i j}$ & Region \\
\hline $6 \times 10^{-3} \lesssim \sin ^{2} \theta_{i j}$ & Sensitivity range of MiniBoone \\
$4 \times 10^{-4} \lesssim \sin ^{2} \theta_{i j}$ & Maximal effect of shock \\
$4 \times 10^{-6} \lesssim \sin ^{2} \theta_{i j} \lesssim 4 \times 10^{-4}$ & Transition region \\
$\sin ^{2} \theta_{i j} \lesssim 4 \times 10^{-6}$ & No effect of shock \\
\hline
\end{tabular}

Table 3: The effect of the shock wave for the parameter space of $\sin ^{2} \theta_{i j}$ with $i=1,2$ and $\mathbf{j}=3-5$.

unchanged. For the cases of N2+I3 and $\mathrm{H} 2+\mathrm{N} 3$ mass hierarchies $F_{\text {noshock }}$ is scaled as $F_{\text {noshock }} \rightarrow k^{2} F_{\text {noshock }}$. As a result the flux and therefore number of observed events before and after the propagation of a shock wave scale as $k^{2}$, the statistical uncertainties scale as $k^{-1}$ and the average energies remain unchanged. Therefore for $k<1$ the number of events decreases, and the uncertainties in the number of events and average energies increases. As the shock wave passes through the resonance the flux is $F_{e} \simeq F_{\text {shock}}$, which is independent of $k$. Therefore the total number of events is approximately unchanged. However, as the shock passes through a resonance the number of events increases, these corresponds to the lowest energy neutrinos, as a result the average energy decreases. For smaller $k$ the relative increase in the number of events is larger and therefore the decrease in the average energy is larger. At later times there is an increase in the average energy as the resonance condition is satisfied for higher energy neutrinos, for smaller $k$ the relative increase is larger and therefore the increase in the average energy is larger. As a result the structure of the average energy plot remains but is stretched for smaller $k$. Simulations show that the number of events during the shock propagation is approximately independent of $k$ for $k \lesssim 1$ and the average energy plot is stretched as described above.

\subsection{Three active neutrinos}

We first consider the case of a "standard" supernova at $10 \mathrm{kpc}$ from earth. If the mass hierarchy of the neutrinos is inverted, the flux of anti-electron neutrinos in the detector is given by

$$
F_{e}=F_{x}^{0}+\bar{P}_{e e}\left(F_{e}^{0}-F_{x}^{0}\right)
$$

where $\bar{P}_{e e}$ is given by Eq. (15). For the values of $\sin ^{2} \theta_{13} \gtrsim 10^{-3}$ that we assume throughout this paper, $P_{13} \simeq 0$ in the absence of shock and the flux of $\bar{\nu}_{e}$ at the detector $F_{e} \simeq F_{x}^{0}$. As the shock crosses the resonance density, $P_{13} \simeq 1$ as discussed in section 2.2 and the flux of $\bar{\nu}_{e}$ at the detector is given by $F_{e} \simeq\left(1-\left|U_{e 1}\right|^{2}\right) F_{x}^{0}+\left|U_{e 1}\right|^{2} F_{e}^{0}$. If the average energy of $\bar{\nu}_{x}$ is larger than that of $\bar{\nu}_{e}$ as obtained in the LL simulations, the average energy will decrease as a result of the shock effect. This can be seen from Fig. 3(a), where we show the time evolution of the average energy of the neutrinos detected in a megaton water Cerenkov detector. The band shows the statistical error expected in the measured average energy. For no shock (NS) the average energy remains almost constant with time. On the other hand the average energy decreases and hence shows "bumps" in the time profile as the shock crosses the position of the $\Delta m_{31}^{2}$ driven resonance. We get a single bump for the forward shock only and double bump when the reverse shock is also present. This is due to the shape of the flip probability shown in Figs. 10and 2] Also shown (in Fig. 3(c) and Fig. 3(e)) is what is expected if the difference between the average energies of the $\bar{\nu}_{e}$ and $\nu_{x}$ created in the supernova were not so well separated. We notice that even though the effect of the shock is less dramatic, nonetheless it is still there and should be observable. Particularly note that for G2 the average energy of $\bar{\nu}_{e}$ and $\bar{\nu}_{x}$ are equal, therefore the change in the average energy measured by the detector is due to the change in the number of detected neutrinos. In Fig. 4 we show the total number of events expected for the LL (panel (a)), G1 (panel (c)) and G2 (panel (e)) cases, with and without the shock effects. Since the cross section for the detection cross section increases quadratically with energy, the number of events will decrease when the shock crosses the $\Delta m_{31}^{2}$ resonance density and this results in lowering of the number of events and hence again gives single bump and double bump for the forward and forward+reverse shock cases respectively, as a function of time.

The corresponding results taking into account the effects of turbulence are shown in right-hand panels of Figs. 3 and 4. Panels (b), (d) and (f) in these figures correspond to the LL, G1 and G2 simulations respectively. The inclusion of turbulence changes the signal for $t \gtrsim 5 \mathrm{~s}$. This is when the resonant density is in the turbulent region. As discussed before, the system is largely depolarized giving $P_{13} \simeq 1 / 2$. The flux at the detector when 


\begin{tabular}{|c|c|c|}
\hline Hierarchy & $F_{\text {noshock }}$ & $F_{\text {shock }}$ \\
\hline $\mathrm{N} 2+\mathrm{N} 3$ & $\left|U_{e 1}\right|^{2} F_{e}^{0}$ & $P_{24} P_{25}\left|U_{e 2}\right|^{2} F_{x}^{0}$ \\
\hline $\mathrm{N} 2+\mathrm{I} 3$ & $\left|U_{e 3}\right|^{2} F_{e}^{0}+\left(\left|U_{e 4}\right|^{2}+\left|U_{e 5}\right|^{2}\right) F_{x}^{0}$ & $\begin{array}{l}\left|U_{e 1}\right|^{2} P_{13} F_{e}^{0}+\left(\left(P_{24}+P_{25}\right)\left|U_{e 2}\right|^{2}\right. \\
\left.\quad+P_{24} P_{25}\left(\left|U_{e 1}\right|^{2}-\left|U_{e 2}\right|^{2}\right)\right) F_{x}^{0}\end{array}$ \\
\hline $\mathrm{H} 2+\mathrm{N} 3$ & $\left|U_{e 4}\right|^{2} F_{e}^{0}+\left(\left|U_{e 3}\right|^{2}+\left|U_{e 5}\right|^{2}\right) F_{x}^{0}$ & $P_{24}\left|U_{e 1}\right|^{2} F_{e}^{0}+P_{25}\left|U_{e 2}\right|^{2} F_{x}^{0}$ \\
\hline $\mathrm{H} 2+\mathrm{I} 3$ & $\left|U_{e 2}\right|^{2} F_{x}^{0}$ & $P_{25}\left|U_{e 1}\right|^{2} F_{x}^{0}$ \\
\hline $\mathrm{I} 2+\mathrm{N} 3$ & $\left|U_{e 2}\right|^{2} F_{x}^{0}$ & $P_{24} P_{25}\left|U_{e 1}\right|^{2} F_{e}^{0}$ \\
\hline $\mathrm{I} 2+\mathrm{I} 3$ & $\left(\left|U_{e 1}\right|^{2}+\left|U_{e 2}\right|^{2}\right) F_{x}^{0}$ & $-P_{13}\left|U_{e 1}\right|^{2} F_{x}^{0}$ \\
\hline
\end{tabular}

Table 4: The flux of neutrinos in the approximation that $\left|U_{e 1}\right|^{2},\left|U_{e 2}\right|^{2}>>\left|U_{e 3}\right|^{2},\left|U_{e 4}\right|^{2},\left|U_{e 5}\right|^{2}$ and $P_{1 i}, P_{3 i} \simeq$ $P_{2 i}$, where $\mathrm{i}=4$ or 5 . The total flux in presence of shock is given by $F_{e}=F_{\text {noshock }}+F_{\text {shock }}$, where $F_{\text {noshock }}=F_{e}$ without the shock and $F_{\text {shock }}$ is the extra component due to the shock effect.

the shock crosses the resonance region is now given by $F_{e} \simeq\left(1-0.5\left|U_{e 1}\right|^{2}\right) F_{x}^{0}+0.5\left|U_{e 1}\right|^{2} F_{e}^{0}$. For the same arguments as before, the average energy is lower than the case of no shock, however the effect now is much less than the case where turbulence was not considered. Therefore, the single and double bump features expected from shock effects get smeared out to a large extent. However, we still see a non-trivial variation of the expected average energy in the detector as a function of time, both for the LL and G1/G2 simulations.

\subsection{Three active and two sterile neutrinos}

For the case where we have two extra light sterile neutrinos in addition to the three active ones, the situation gets a lot more involved due to the possibility of multiple resonances as discussed in section 2.1. The effect of the shock is also richer here since the shock passes through the different resonance densities at different times. In Table 4 the resultant neutrino flux at the detector is given in terms of the original neutrino fluxes produced, for the different neutrino mass spectra considered, for both with and without shock effects. We reiterate that we have chosen mixing angles such that in absence of shock effects all resonances are adiabatic. The effect of the shock is to turn the adiabatic resonant transition into non-adiabatic ones, as discussed before. For the case where sterile neutrinos are present, the change in number of events are characterized by the oscillations of active neutrinos into sterile species and vice-versa. Thus in this case, difference in the initial neutrino energy spectra is not a pre-requisite for observing resonant oscillations and shock effects, unlike in the case of 3 active neutrinos only. As the shock moves in time, its effect is imprinted on the time dependence of the neutrino signal, as in the case of 3 active neutrinos only. However, with sterile neutrinos there are further modulations in the signal because the shock passes through the additional sterile resonances. The number of resonances is dependent on the number of sterile neutrinos as well as the neutrino mass spectrum and therefore the expected signal is often different for each scenario, as described in detail in [19. In fact, as we will see, even when the shock passes through the $\Delta m_{31}^{2}$ driven active resonance, the time profile and energy spectrum of the observed neutrinos in many of the possible mass spectrum listed in Eqs. (17)-(22), are different than what is expected for the case of 3 active neutrinos only. Typically, the shock crosses the multiple "sterile resonances" at very early times $(t \lesssim 2$ $\mathrm{sec})$, while it crosses the $\Delta m_{31}^{2}$ driven "active resonance" at later times ( $\left.t \gtrsim 3 \mathrm{sec}\right)$. Therefore in what follows, we will present results separately at late and early times to show clearly the effect of the shock wave on the neutrino signal in the detector. For later times, we will consider time bins of $100 \mathrm{~ms}$, while at earlier times since the time dependence is much more sharp, we present our results for smaller time bins of $10 \mathrm{~ms}$.

\subsection{Neutrino signal at late times}

We begin by discussing the evolution of the expected neutrino event rate and average energy at later times. The total number of events for the different mass spectra are shown in Fig. 5 while the expected average energies 
are shown in Fig. 6, at times between $t=3-12 \mathrm{~s}$. These plots show the impact on the signal when the shock wave passes through the $\Delta m_{31}^{2}$ resonance between active neutrinos. In panels (a)-(d) of Figs. 5 and 6 we show only the mass spectrum cases where $\Delta m_{31}^{2}<0$, in which we have resonance and hence also shock effects. Which mass spectra will get the shock effects can be easily seen from Table 4. Since at late times the resonance that gets affected by the shock is the $\Delta m_{31}^{2}$ driven resonance between the 1-3 states, the relevant jump probability involved is $P_{13}$. All other jump probabilities are zero here, from our choice of the mixing angles. We can see from Table 4 that the N2+I3 and I2+I3 are the only cases which will get affected. For all the other mass spectra possible, we do not expect any modulation in the signal at late times due to shock effects. Hence we show only the signal for the $\mathrm{N} 2+\mathrm{I} 3$ and $\mathrm{I} 2+\mathrm{I} 3$ cases. For comparison we show in the last 2 panels of these figures the case of only active neutrinos (I3).

In the approximation that $\left|U_{e 3}\right|^{2},\left|U_{e 4}\right|^{2}$ and $\left|U_{e 5}\right|^{2}$ can be neglected in comparison to $\left|U_{e 1}\right|^{2}$ and $\left|U_{e 2}\right|^{2}$, we note from Table 4 that in absence of shock,

$$
\begin{aligned}
I 3 & \Rightarrow F_{e} \simeq F_{x}^{0}, \\
N 2+I 3 & \Rightarrow F_{e} \simeq 0, \\
I 2+I 3 & \Rightarrow F_{e} \simeq F_{x}^{0},
\end{aligned}
$$

whereas when the shock passes through the 1-3 resonance the fluxes are modified to,

$$
\begin{aligned}
I 3 & \Rightarrow F_{e} \simeq F_{x}^{0}\left(1-P_{13}\left|U_{e 1}\right|^{2}\right)+P_{13}\left|U_{e 1}\right|^{2} F_{e}^{0}, \\
N 2+I 3 & \Rightarrow F_{e} \simeq\left|U_{e 1}\right|^{2} P_{13} F_{e}^{0} \\
I 2+I 3 & \Rightarrow F_{e} \simeq\left(1-P_{13}\left|U_{e 1}\right|^{2}\right) F_{x}^{0} .
\end{aligned}
$$

By comparing Eqs. (400)-(42) we note that in absence of shock, while the fluxes are same for I3 and I2+I3 spectra, N2+I3 predicts almost zero fluxes. The black bands in Fig. 5 corroborate the above statement. When the shock passes through the $\Delta m_{31}^{2}$ resonance it increases the $\bar{\nu}_{e}$ flux for $\mathrm{N} 2+\mathrm{I} 3$, while it decreases the same for $\mathrm{I} 2+\mathrm{I} 3$, as can be seen by comparing Eqs. (41) and (42) with Eqs. (44) and (45). In the case of I3, the resultant flux is an admixture of a fraction of the initial $\bar{\nu}_{e}$ and $\nu_{x}$ fluxes. However, since the average energy of initial $\bar{\nu}_{e}$ flux was smaller, the total number of events goes down in the detector when the shock passes through the 1-3 resonance even in this case. We stress that the sterile cases are qualitatively different from the I3 case, since for them the net number flux sees a big increase or decrease due to shock. Therefore, while for I3, the effect of the shock wave comes predominantly through the difference in the average energy of $\bar{\nu}_{e}$ and $\nu_{x}$, for sterile cases we see a combined effect coming from a direct change in the number of neutrinos arriving on earth as well as the difference in the energy spectra of the different species.

For all the mass spectra shown, under the assumption that there is no turbulence, typically a single bump is observed for a forward shock and a double bump for a forward and reverse shock, as expected from the shape of the flip probability shown in Figs. 1 and 2. The effect of taking the turbulence into account is to smear these sharp changes in the oscillation probability due to the effective depolarizing of the resonances. With turbulence, a single bump is observed, followed by a region in which the number of events are typically different from what is expected for no turbulence. This can be seen in the right-hand panels in Fig. 5 ,

The expected average energies for the $\mathrm{N} 2+\mathrm{I} 3$ and $\mathrm{I} 2+\mathrm{I} 3$ cases also show a very striking evolution with time at $t \gtrsim 3 \mathrm{~s}$, which is very different from that predicted by I3. In the case of I3, the average energy decreases in presence of shock as expected, since the shock reduces the conversion probability of the high energy $\nu_{x}$ into $\bar{\nu}_{e}$, thereby decreasing the average energy. Thus it usually shows 1 sharp decrease for forward shock and 2 sharp falls for forward+reverse shock. On the other hand both $\mathrm{N} 2+\mathrm{I} 3$ and $\mathrm{I} 2+\mathrm{I} 3$ predict that the average energy fluctuates on both sides of the average energy expected in absence of shock. The key issue to note here is that the position of resonance is determined by the energy of the neutrino. The higher (lower) energy neutrinos go through the resonance at lower (higher) density. Since the shock moves from higher to lower densities in time, the lower energy neutrinos are affected by the shock earlier than the higher energy ones. For the sterile cases, the effect is more subtle. Here the time evolution of the average energy is a combined effect of the change in the total flux as well as the energy dependence of the resonance position. For the I2+I3 case since resonance happens for lowest energy neutrinos first, the effect of the shock is to reduce them in the flux (cf. Eq.(42) and (45)), thereby increasing the average energy. Eventually, the shock goes through higher energy resonances, and 
this then reduces the average energy. For the N2+I3 case also the resonance happens for the lowest energy neutrinos first, but now the shock effect increases them in the flux and thereby decreases the average energy. Eventually, the shock goes through the high energy resonances, increasing the average energy.

The right-hand panels of Fig. 6 show the average energy after turbulence is taken into consideration. The presence of turbulence has a typical effect on the time evolution of both the total number of events and the average energy deposited by the (anti)neutrinos. One can see that without turbulence both the average energy and number of events after the passage of (both) shock(s) go back to its pre-shock value. This is because the $\bar{P}_{e e}$ before and after the shock are exactly the same in this case. However, since the turbulence changes the flip probability permanently to $P_{i j}$ behind the shock, the number of events and average energy are consistently lower once the shock crosses the resonance point.

\subsection{Neutrino signal at early times}

In Figs. 7 and 9 we show the number of predicted events and average energies as a function of time (in absence of turbulence) for the 5 different mass spectra, between $t=0.1-1.5 \mathrm{~s}$, in short time bins of $10 \mathrm{~ms}$. Figs. 8 and 10 show the corresponding results when turbulence is taken into account. We do not show results for the I2+I3 spectra here since, as can be seen from Table 4 , it depends only on $P_{13}$ which will have shock effects only at the $\Delta m_{31}^{2}$ resonance at late times.

As in the previous subsection, Fig. 7 can be understood in terms of the flux predictions in the presence and absence of the shock, given in Table 4. The only difference is that at early times the shock passes through the sterile resonances and hence we expect contributions coming from the jump probabilities associated with the sterile resonances. In particular, we note that in the absence of shock, the predicted flux on earth is almost zero for $\mathrm{N} 2+\mathrm{I} 3$ and $\mathrm{H} 2+\mathrm{N} 3$, while for $\mathrm{H} 2+\mathrm{I} 3$ and $\mathrm{I} 2+\mathrm{N} 3$ we expect the flux to be $\left|U_{e 2}\right|^{2} F_{x}^{0}$ and for $\mathrm{N} 2+\mathrm{N} 3$ it is predicted to be $\left|U_{e 1}\right|^{2} F_{x}^{0}$. Since $\left|U_{e 1}\right|^{2}>\left|U_{e 2}\right|^{2}$, the expected signal is larger for N2+N3. These features are evident from Fig. 7. As a result of the shock we see a modulation in the resultant signal visible as bumps in the figure. Note that in all the 5 cases shown, the shock effect increases the number of events. The shock powered modulations get affected when turbulence is taken into account and the corresponding results are shown in Fig. 8 .

Fig. 9 shows how the average energy evolves at early times. As we had seen at later times, the effect of the shock wave is to make the average energy fluctuate on both sides of the corresponding static density case. The reason for the initial average energy decrease and subsequent increase is also the same as that discussed in the previous subsection. Fig. 10] shows the average energy evolution when the effect of turbulence is considered. The rapid fluctuations are mellowed due to the presence of turbulence, however we still see statistically significant fluctuations in the average energy at very early times, a feature which, if observed, would provide an almost model independent signal of the presence of extra sterile neutrino species which are mixed mildly with the active neutrinos.

As the water Cerenkov detector can measure the energy of the incoming neutrino rather efficiently, the events can be binned in energy as well as time. In Fig. 11 we present the number of events expected in energy bins of width $10 \mathrm{MeV}$ and time bins of $10 \mathrm{~ms}$. We show results for the $\mathrm{N} 2+\mathrm{I} 3$ spectrum only as an example. This figure is shown for only early times to illustrate the effect of the sterile resonances. As the resonance densities are energy dependent, the propagation of the shock wave can be observed when the events are binned in energy and time. The shock wave crosses the resonant density corresponding to neutrinos with lower energies first, therefore the characteristic 'bumps' are observed in the lower energy bins first. The presence of such energy and time dependent bumps in the resultant signal in the detector provides a 'smoking gun' signal for sterile neutrinos.

\section{Comparison and Discussions}

In Table 5 we show the model independent characteristic features in the expected signal for the eight different cases considered in this paper, the two only active cases (N3 and I3) and the six active plus sterile cases. The time interval is divided into early $(t \lesssim 2 \mathrm{~s})$ and late $(t \gtrsim 3 \mathrm{~s})$ times, as before. For each case we state if we expect large (L) or very small (S) number of events in the 2 time zones. We also indicate in the table whether 


\begin{tabular}{|c|c|c|c|c|}
\hline \multirow{2}{*}{ Spectrum } & \multicolumn{2}{|c|}{ Early Times } & \multicolumn{2}{c|}{ Late Times } \\
\cline { 2 - 5 } & Events & Shock Effect & Events & Shock Effects \\
\hline \hline N3 & L & $\times$ & L & $\times$ \\
I3 & L & $\times$ & L & $\checkmark$ \\
N2+N3 & L & $\checkmark$ & L & $\times$ \\
N2+I3 & S & $\checkmark$ & S & $\checkmark$ \\
H2+N3 & S & $\checkmark$ & S & $\times$ \\
H2+I3 & L & $\checkmark$ & L & $\times$ \\
I2+N3 & L & $\checkmark$ & L & $\times$ \\
I2+I3 & L & $\times$ & L & $\checkmark$ \\
\hline
\end{tabular}

Table 5: The expected trends in the neutrino signal in a megaton water Cerenkov detector at early and late times. The symbols L and S signify large and small number of events respectively. The presence or absence of shock effects are shown by the $\checkmark$ and $\times$ symbols.

we expect shock induced sharp modulations in the signal or not for each of the possible mass spectra. By comparing the predictions for all the cases, we can infer the following:

- 1. If large events are seen at early times,

2. shock effects are not seen at early times,

3. a large number of events are seen at late times and

4. shock effects are not seen at late times

then we must have the N3 spectrum.

- 1. If very few events are seen at early times,

2. shock effects are seen at early times,

3. very few events are seen at late times and

4. shock effects are seen at late times

then we must have the $\mathrm{N} 2+\mathrm{I} 3$ spectrum.

- 1. If very few events are seen at early times,

2. shock effects are seen at early times,

3. very few events are seen at late times and

4. shock effects are not seen at late times

then we must have the $\mathrm{H} 2+\mathrm{N} 3$ spectrum.

- 1. If a large number of events are seen at early times,

2. shock effects are seen at early times,

3. a large number of events are seen at late times and

4. shock effects are not seen at late times

then we could have either $\mathrm{N} 2+\mathrm{N} 3$ or $\mathrm{H} 2+\mathrm{I} 3$ or $\mathrm{I} 2+\mathrm{N} 3$ spectrum.

- 1. If a large number of events are seen at early times,

2. shock effects are not seen at early times,

3. a large number of events are seen at late times and 


\section{4. shock effects are seen at late times}

then we could have either I3 or I2+I3 spectrum. This degeneracy can be split by measurng the energy dependence (c.f. Figure 6 (c) and (d)).

Therefore, from the Table 5 we conclude that, irrespective of model uncertainties, the presence of sterile neutrinos can be easily proved from modulation of the signal due to shock effects at early time. Only the I2+I3 case does not give any time modulation, even though the sterile neutrinos are present in this case. In addition, from the point-wise discussion above we can see how well we can distinguish one mass spectrum from the other. $\mathrm{N} 3, \mathrm{~N} 2+\mathrm{I} 3$ and $\mathrm{H} 2+\mathrm{N} 3$ can be uniquely determined by comparing early time behavior of the signal with its late time behavior. The $\mathrm{N} 2+\mathrm{N} 3, \mathrm{H} 2+\mathrm{I} 3$ and $\mathrm{I} 2+\mathrm{N} 3$ can be separated from the rest, but since they predict similar trends at early and late times, one will be need a more careful model dependent study to unambiguously disentangle them from each other. Similarly, though I3 and I2+I3 can be separated from the other spectra, one will need a more careful analysis to distinguish between the two.

\section{Summary and Conclusions}

Model independent information about the neutrino mass spectrum can be obtained through observation of signatures of supernova shock wave(s) on the resultant neutrino signal in terrestrial detectors. In particular, such signals probe the existence of extra sterile neutrino through their possible resonant transition with active neutrinos inside the supernova. Oscillations between sterile neutrino and active ones are characterized by unique signatures in the final neutrino energy spectrum as well as their evolution with time. While the impact of the presence of sterile neutrinos on the time evolution of the neutrino is due to shock effects, their impact on the resultant neutrino flux and spectra comes from neutrino oscillations both with and without shock effects.

Concerns had been raised recently over the observability of the shock effects due to the turbulent density variations following the shock wave which prove to be very important. In this paper we made a detailed study of the turbulent shock effects on the neutrino induced galactic supernova signal expected in megaton water Cerenkov detector. Water detectors can give information on the number, the energy spectrum, as well the time profile of the arriving (anti)neutrinos. We have shown that the impact of the supernova shock waves is evident in the neutrino signature in megaton water Čerenkov detectors in all of these, making this class of detectors extremely good for studying shock effects. We have considered the impact of the turbulence left behind by the shock wave and have seen that although the shock effect is diluted, it is still significant and we still expect to observe them in the megaton class of detectors. We have concentrated on the discernable signatures of sterile neutrinos, which might be mixed mildly with the 3 active neutrinos. We have illustrated the effects using sterile neutrino parameters which were chosen in a fit to all neutrino data including LSND. However we showed that the observable signals persist for much smaller mixing angles than are observable by the LSND (or MiniBOONE) experiments. Hence our results are relevant whatever the outcome of the MiniBOONE data.

The most striking evidence for sterile neutrinos in the supernova neutrino signal are sharp bumps at $t \lesssim 1$ secin the observed number flux as well as the average energy of the $\bar{\nu}_{e}$ detected through their capture on protons in megaton water detectors. These can be caused only by sterile neutrino resonances inside the supernova. Only the $\mathrm{I} 2+\mathrm{I} 3$ case for the mass spectrum does not predict these early time shock induced modulations in the signal. In addition, a model independent comparison of the signal trend between early and late times can give us a rather unambiguous signature on 3 of the 8 possible mass spectra considered in this paper. The N3, N2+I3 and $\mathrm{H} 2+\mathrm{N} 3$ cases predict unique combination of behavior at early and late times and hence can be determined model independently from the observations. The remaining 5 cases can be classed into 2 categories depending on their combination of predicted trends at early and late times. Distinguishing the I3 from the I $2+\mathrm{I} 3$ spectra would require a more careful model dependent analysis of the future supernova neutrino data. Similarly, the $\mathrm{N} 2+\mathrm{N} 3, \mathrm{H} 2+\mathrm{I} 3$ and $\mathrm{I} 2+\mathrm{N} 3$ can be separated from the relative differences in their predictions of average energy and number of events as a function of time.

One important byproduct of the turbulent effects involving active-sterile neutrino mixing is that, even if the energy spectrum and luminosisites of the active neutrinos are initially the same, the depolarising effect of the turbulence for the active sterile resonances in the wake of the shock front will make the active neutrino spectra spectra and luminosities significantly different. As a result the atmospheric neutrino resonant effects involving 
the active neutrinos in the presence of sterile neutrinos may be expected to give rise to more significant effects in the supernova neutrino signal than is the case without sterile neutrinos.

\section{Acknowledgment}

We thank T. Kajita for discussion on water Čerenkov detectors. SC wishes to thank PPARC and the University of Oxford for financial support during this work.

\section{References}

[1] R. M. Bionta et al., Phys. Rev. Lett. 58 (1987) 1494.

[2] K. Hirata et al. [KAMIOKANDE-II Collaboration], Phys. Rev. Lett. 58 (1987) 1490.

[3] H. A. Bethe, Astrophys. J. 412, 192 (1993). V. Barger, D. Marfatia and B. P. Wood, Phys. Lett. B 532, 19 (2002); H. Minakata and H. Nunokawa, Phys. Lett. B 504, 301 (2001); C. Lunardini and A. Y. Smirnov, Astropart. Phys. 21, 703 (2004); A. Mirizzi and G. G. Raffelt, Phys. Rev. D 72, 063001 (2005).

[4] B. T. Cleveland et al., Astrophys. J. 496, 505 (1998); J. N. Abdurashitov et al. [SAGE Collaboration], J. Exp. Theor. Phys. 95, 181 (2002); [Zh. Eksp. Teor. Fiz. 122, 211 (2002)] W. Hampel et al. [GALLEX Collaboration], Phys. Lett. B 447, 127 (1999); C. Cattadori, Talk at Neutrino 2004, Paris, France, June 1419, 2004; S. Fukuda et al. [Super-Kamiokande Collaboration], Phys. Lett. B 539, 179 (2002); B. Aharmim et al. [SNO Collaboration], Phys. Rev. C 72, 055502 (2005).

[5] T. Araki et al. [KamLAND Collaboration], Phys. Rev. Lett. 94, 081801 (2005).

[6] M. Apollonio et al., Eur. Phys. J. C 27, 331 (2003); F. Boehm et al., Phys. Rev. D 64, 112001 (2001).

[7] Y. Ashie et al. [Super-Kamiokande Collaboration], Phys. Rev. D 71, 112005 (2005).

[8] E. Aliu et al. [K2K Collaboration], Phys. Rev. Lett. 94, 081802 (2005).

[9] D. G. Michael et al. [MINOS Collaboration], Phys. Rev. Lett. 97, 191801 (2006).

[10] M. Maltoni et al., New J. Phys. 6, 122 (2004), hep-ph/0405172 v5; S. Choubey, arXiv:hep-ph/0509217; S. Goswami, Int. J. Mod. Phys. A 21, 1901 (2006); A. Bandyopadhyay et al., Phys. Lett. B 608, 115 (2005); G. L. Fogli et al., Prog. Part. Nucl. Phys. 57, 742 (2006).

[11] K. Anderson et al., arXiv:hep-ex/0402041.

[12] Y. Itow et al., arXiv:hep-ex/0106019.

[13] D. S. Ayres et al. [NOvA Collaboration], arXiv:hep-ex/0503053.

[14] C. Volpe, J. Phys. G 34, R1 (2007); S. K. Agarwalla, S. Choubey and A. Raychaudhuri, arXiv:hep-ph/0610333 and references therein.

[15] C. Albright et al. [Neutrino Factory/Muon Collider Collaboration], arXiv:physics/0411123.

[16] A. S. Dighe and A. Y. Smirnov, Phys. Rev. D 62, 033007 (2000); C. Lunardini and A. Y. Smirnov, JCAP 0306, 009 (2003); C. Lunardini and A. Y. Smirnov, Nucl. Phys. B 616, 307 (2001); S. Choubey, D. Majumdar and K. Kar, J. Phys. G 25, 1001 (1999); G. Dutta et al., Phys. Rev. D 61, 013009 (2000); A. S. Dighe et al., JCAP 0401, 004 (2004); H. Minakata et al., Phys. Lett. B 542, 239 (2002).

[17] A. S. Dighe, M. T. Keil and G. G. Raffelt, JCAP 0306, 006 (2003); A. Bandyopadhyay, S. Choubey, S. Goswami and K. Kar, arXiv:hep-ph/0312315 T. Marrodan Undagoitia, F. von Feilitzsch, M. GogerNeff, K. A. Hochmuth, L. Oberauer, W. Potzel and M. Wurm, J. Phys. Conf. Ser. 39, 287 (2006); S. Skadhauge and R. Z. Funchal, arXiv:hep-ph/0611194. 
[18] A. S. Dighe, M. T. Keil and G. G. Raffelt, JCAP 0306, 005 (2003).

[19] S. Choubey, N. P. Harries, and G. G. Ross Phys. Rev. D 74, 053010 (2006).

[20] S. Hannestad and G. Raffelt, Astrophys. J. 507, 339 (1998); R. Buras et al., Astrophys. J. 587, 320 (2003); M. T. Keil, G. G. Raffelt and H. T. Janka, Astrophys. J. 590, 971 (2003); M. Liebendoerfer et al., Astrophys. J. 620, 840 (2005). G. G. Raffelt, M. T. Keil, R. Buras, H. T. Janka and M. Rampp, arXiv:astro-ph/0303226

[21] K. Takahashi, M. Watanabe, K. Sato and T. Totani, Phys. Rev. D 64, 093004 (2001).

[22] R. C. Stimesrato, G. M. Fuller, arXiv:astro-ph/0205390

[23] G. L. Fogli, E. Lisi, A. Mirizzi and D. Montanino, Phys. Rev. D 68, 033005 (2003).

[24] V. Barger, P. Huber and D. Marfatia, Phys. Lett. B 617, 167 (2005).

[25] R. Tomas, M. Kachelriess, G. Raffelt, A. Dighe, H. T. Janka and L. Scheck, JCAP 0409, 015 (2004).

[26] G. L. Fogli, E. Lisi, A. Mirizzi and D. Montanino, JCAP 0504, 002 (2005).

[27] G. L. Fogli, E. Lisi, A. Mirizzi and D. Montanino, arXiv:hep-ph/0603033.

[28] A. Friedland, and A. Gruizinov arXiv:astro-ph/0607244.

[29] C. Athanassopoulos et al., (The LSND Collaboration) Phys. Rev. Lett. 77, 3082 (1996); C. Athanassopoulos et al., (The LSND Collaboration) Phys. Rev. Lett. 81, 1774 (1998).

[30] J. J. Gomez-Cadenas and M. C. Gonzalez-Garcia, Z. Phys. C 71, 443 (1996); S. Goswami, Phys. Rev. D 55, 2931 (1997). S. M. Bilenky, C. Giunti and W. Grimus, Eur. Phys. J. C 1, 247 (1998).

[31] M. Sorel, J. M. Conrad and M. H. Shaevitz, Phys. Rev. D 70, 073004 (2004).

[32] A.Strumia, "Neutrino masses and mixings and...", http://astrumia.web.cern.ch/astrumia/review.pdf

[33] http://www-boone.fnal.gov/

[34] L. Wolfenstein, Phys. Rev. D 17, 2369 (1978);

[35] S. P. Mikheev and A. Y. Smirnov, Sov. J. Nucl. Phys. 42, 913 (1985) [Yad. Fiz. 42, 1441 (1985)]; S. P. Mikheev and A. Y. Smirnov, Nuovo Cim. C 9, 17 (1986).

[36] V. D. Barger, K. Whisnant, S. Pakvasa and R. J. N. Phillips, Phys. Rev. D 22, 2718 (1980).

[37] E. Lisi, A. Marrone, D. Montanino, A. Palazzo and S. T. Petcov, Phys. Rev. D 63, 093002 (2001); G. L. Fogli, E. Lisi, D. Montanino and A. Palazzo, Phys. Rev. D 65, 073008 (2002); A. Friedland, Phys. Rev. D 64, 013008 (2001).

[38] S. T. Petcov, Phys. Lett. B 200, 373 (1988).

[39] B. Dasgupta and A. Dighe, arXiv:hep-ph/0510219.

[40] T. K. Kuo and J. Pantaleone, Rev. Mod. Phys. 61, 937 (1989); T. K. Kuo and J. Pantaleone, Phys. Rev. D 37, 298 (1988).

[41] K. Kifonidis, T. Plewa, L. Scheck, H. T. Janka, and E. Müller arXiv:astro-ph/0511369.

[42] M. T. Keil, arXiv:astro-ph/0308228; M. T. Keil, G. G. Raffelt and H. T. Janka, Astrophys. J. 590, 971 (2003).

[43] R. Tomas, D. Semikoz, G. G. Raffelt, M. Kachelriess and A. S. Dighe, Phys. Rev. D 68 (2003) 093013

[44] Y. Declais et al., Phys. Lett. B 338, 383 (1994); F. Dydak et al., Phys. Lett. B 134, 281 (1984); I. E. Stockdale et al., Phys. Rev. Lett. 52, 1384 (1984); B. Armbruster et al. [KARMEN Collaboration], Phys. Rev. D 65, 112001 (2002); P. Astier et al. [NOMAD Collaboration], Phys. Lett. B 570, 19 (2003). 

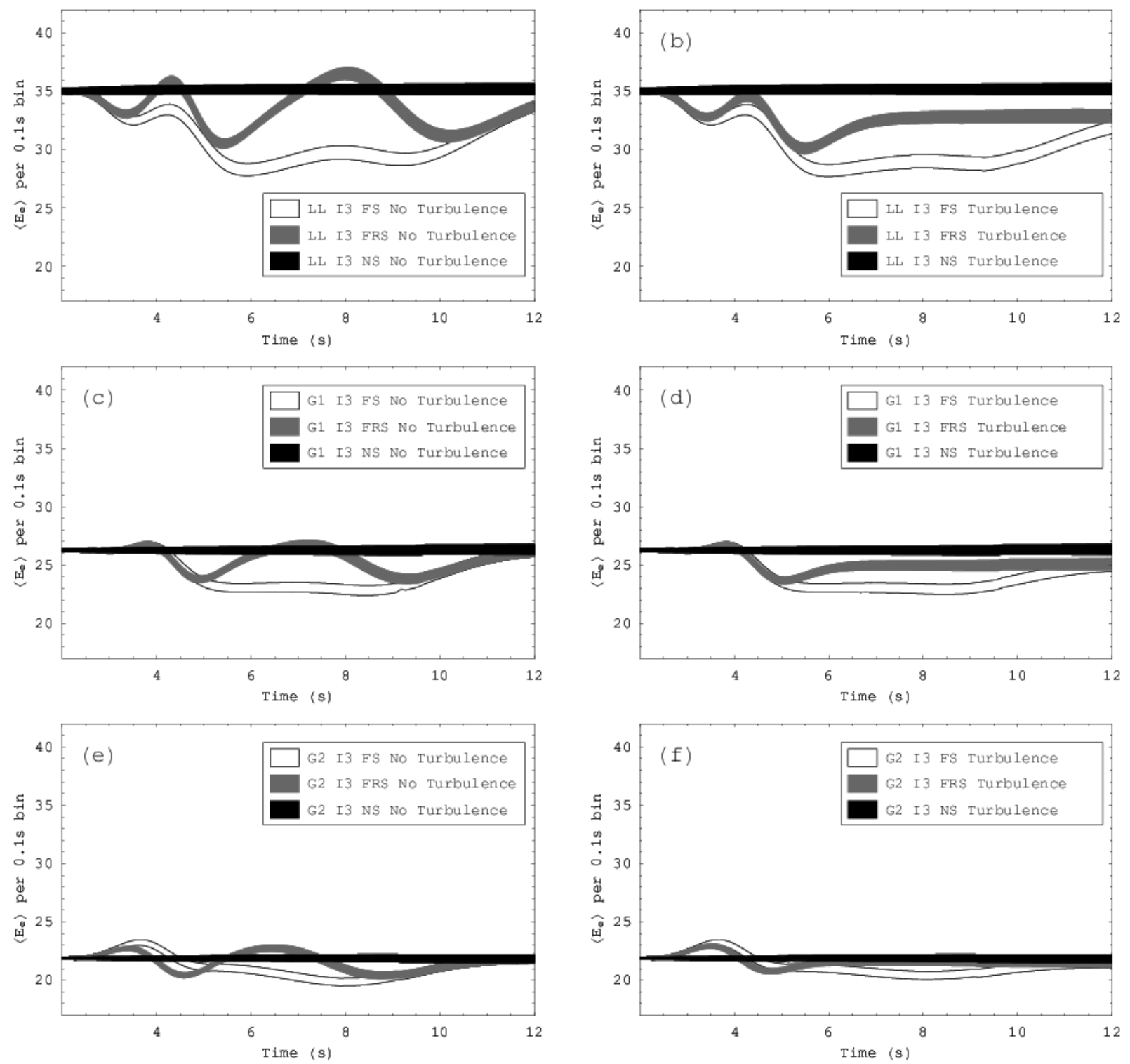

Figure 3: The average energy in 100ms bins, for the cases of a forward shock (FS), a forward and reverse shock (FRS) and no shock (NS): (a)LL no turbulence, (b)LL with Turbulence, (c)G1 no turbulence, (d) G1 with turbulence, (e)G2 no turbulence, (f) G2 with turbulence. 

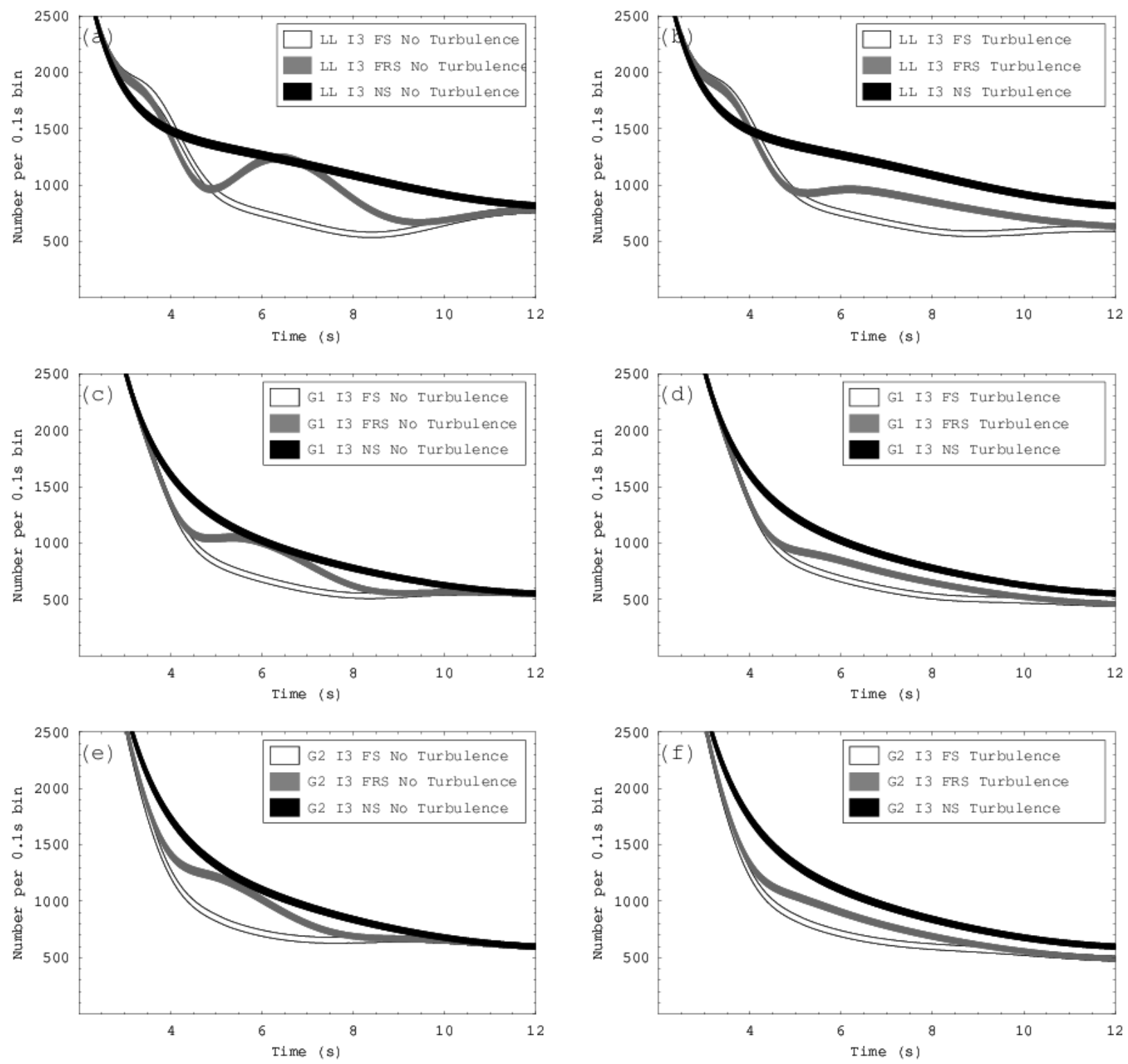

Figure 4: The number of events in $100 \mathrm{~ms}$ bins, for the cases of a forward shock (FS), a forward and reverse shock (FRS) and no shock (NS): (a)LL no turbulence, (b)LL with Turbulence, (c)G1 no turbulence, (d) G1 with turbulence, (e)G2 no turbulence, (f) G2 with turbulence. 

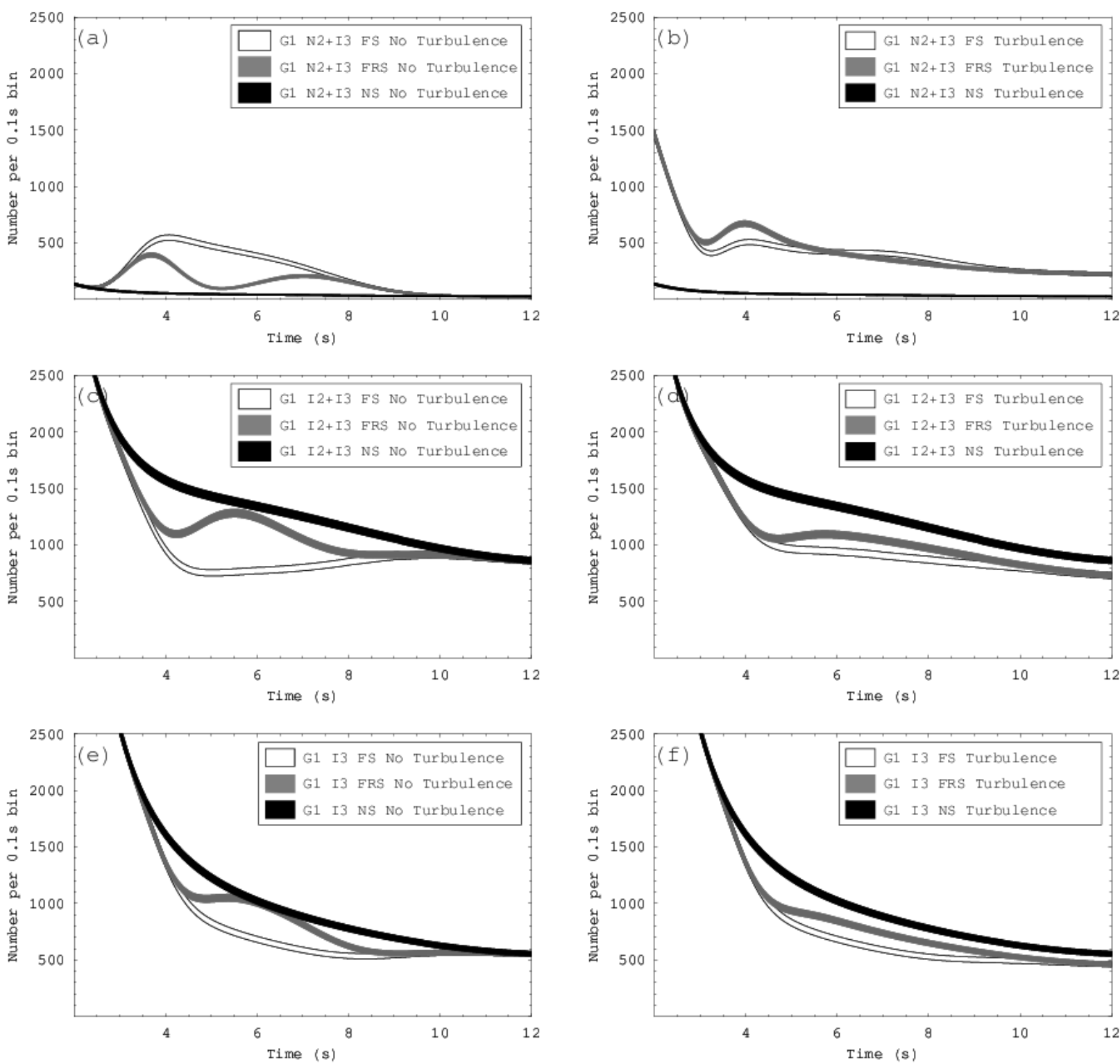

Figure 5: The number of events in $100 \mathrm{~ms}$ bins, for the cases of a forward shock (FS), a forward and reverse shock (FRS) and no shock (NS): (a)N2 + I3 no turbulence, (b)N2 + I3 with turbulence, (c)I2 + I3 no turbulence, (d)I2 + I3 with turbulence, (e)I3 no turbulence, (f)I3 with turbulence. 

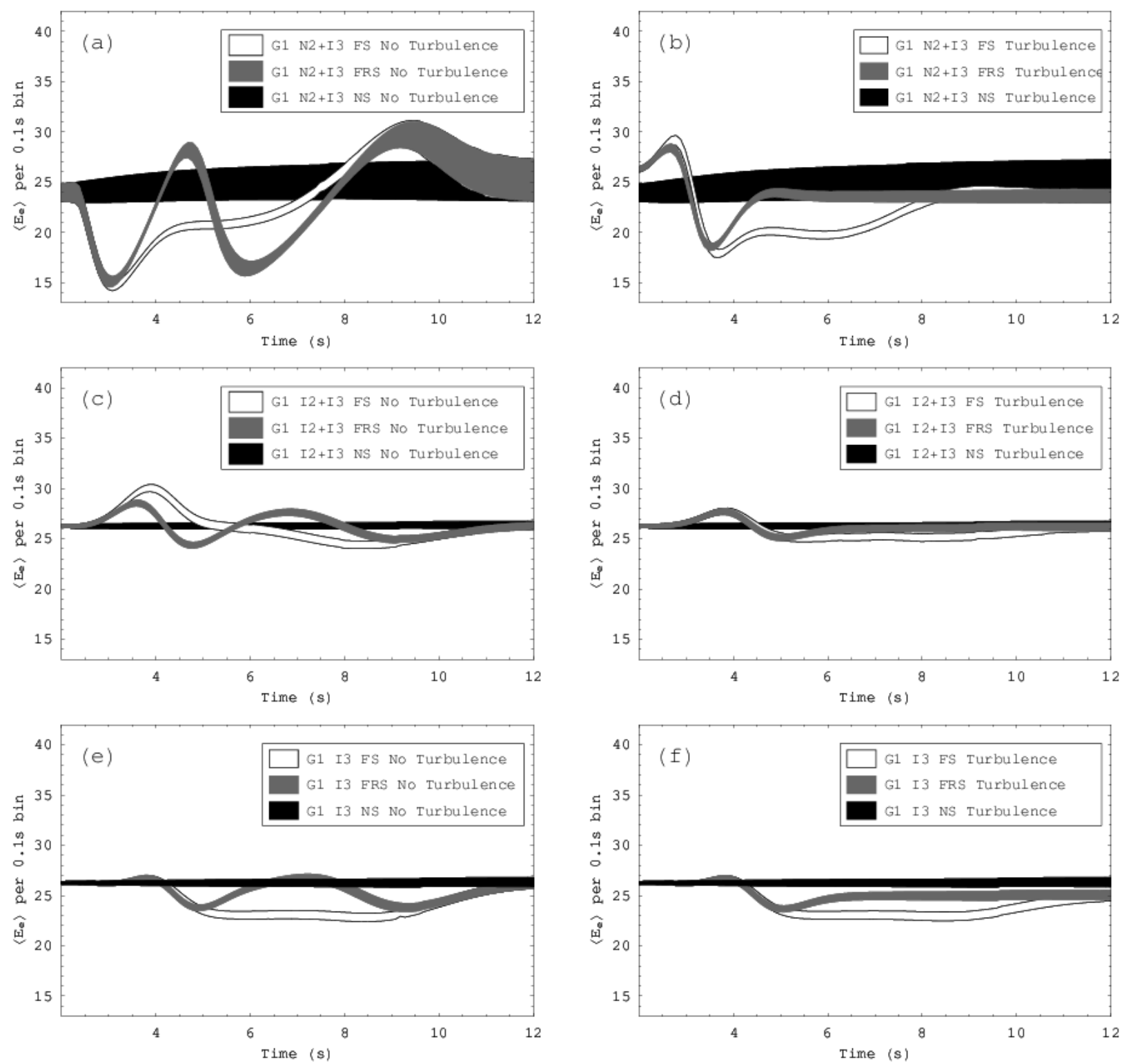

Figure 6: The average energy in $100 \mathrm{~ms}$ bins, for the cases of a forward shock (FS), a forward and reverse shock (FRS) and no shock (NS), with the initial energy spectra G1 as discussed in the text: (a)N2 + I3 no turbulence, (b) $\mathrm{N} 2+\mathrm{I} 3$ with turbulence, (c)I2 + I3 no turbulence, (d)I2 + I3 with turbulence, (e)I3 no turbulence, (f)I3 with turbulence. 

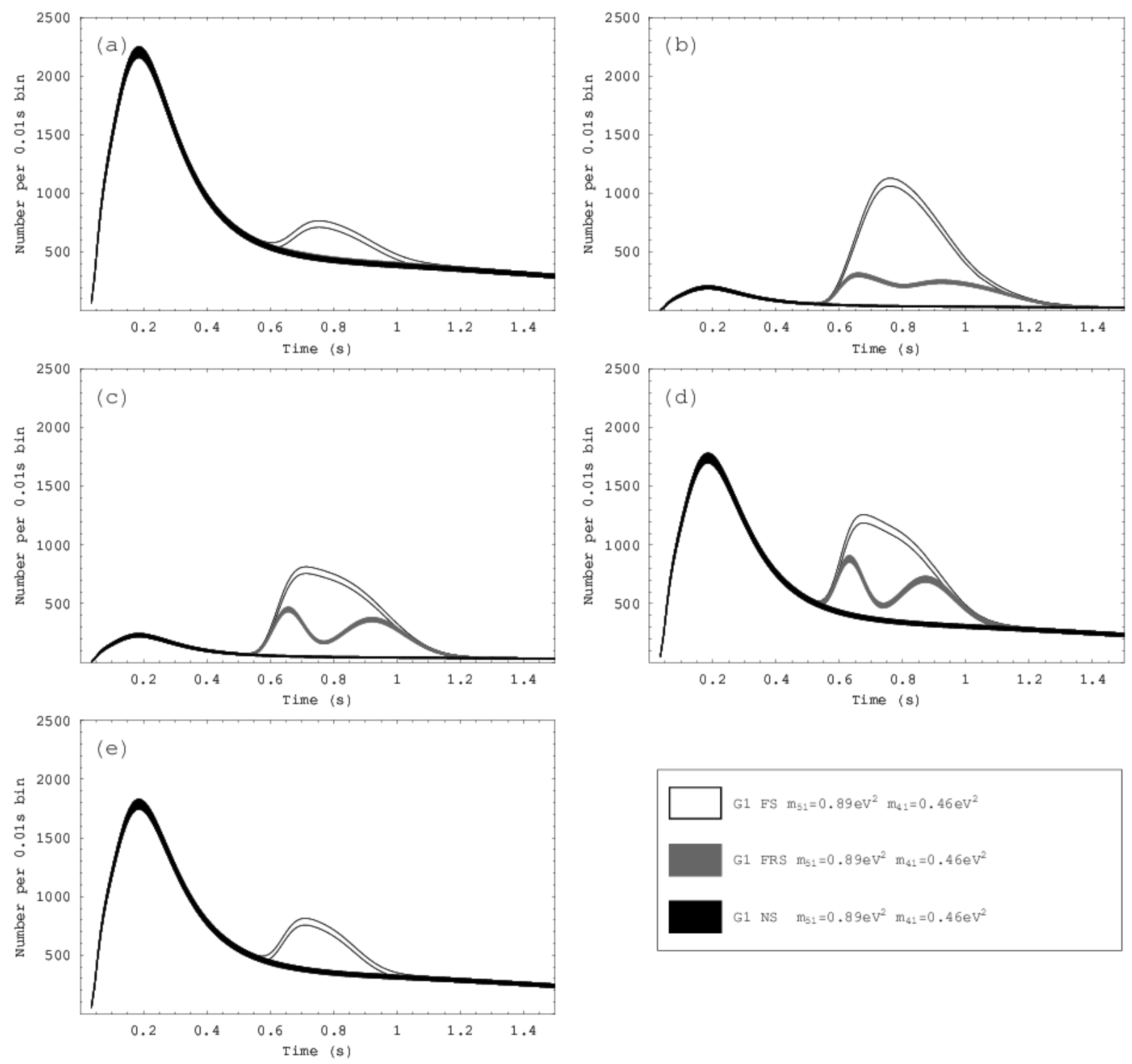

Figure 7: The number of events in $10 \mathrm{~ms}$ bins, for the cases of $\Delta m_{41}^{2}=0.46 \mathrm{MeV}^{2}$ and $\Delta m_{51}^{2}=0.54 \mathrm{MeV}^{2}$, with no turbulence, a forward shock (FS), a forward and reverse shock (FRS) and no shock (NS), with the initial energy spectra G1 as discussed in the text:(a)N2 + N3, (b)N2 + I3, (c)H2 + N3, (d)H2 + I3, (e)I2 + N3. 

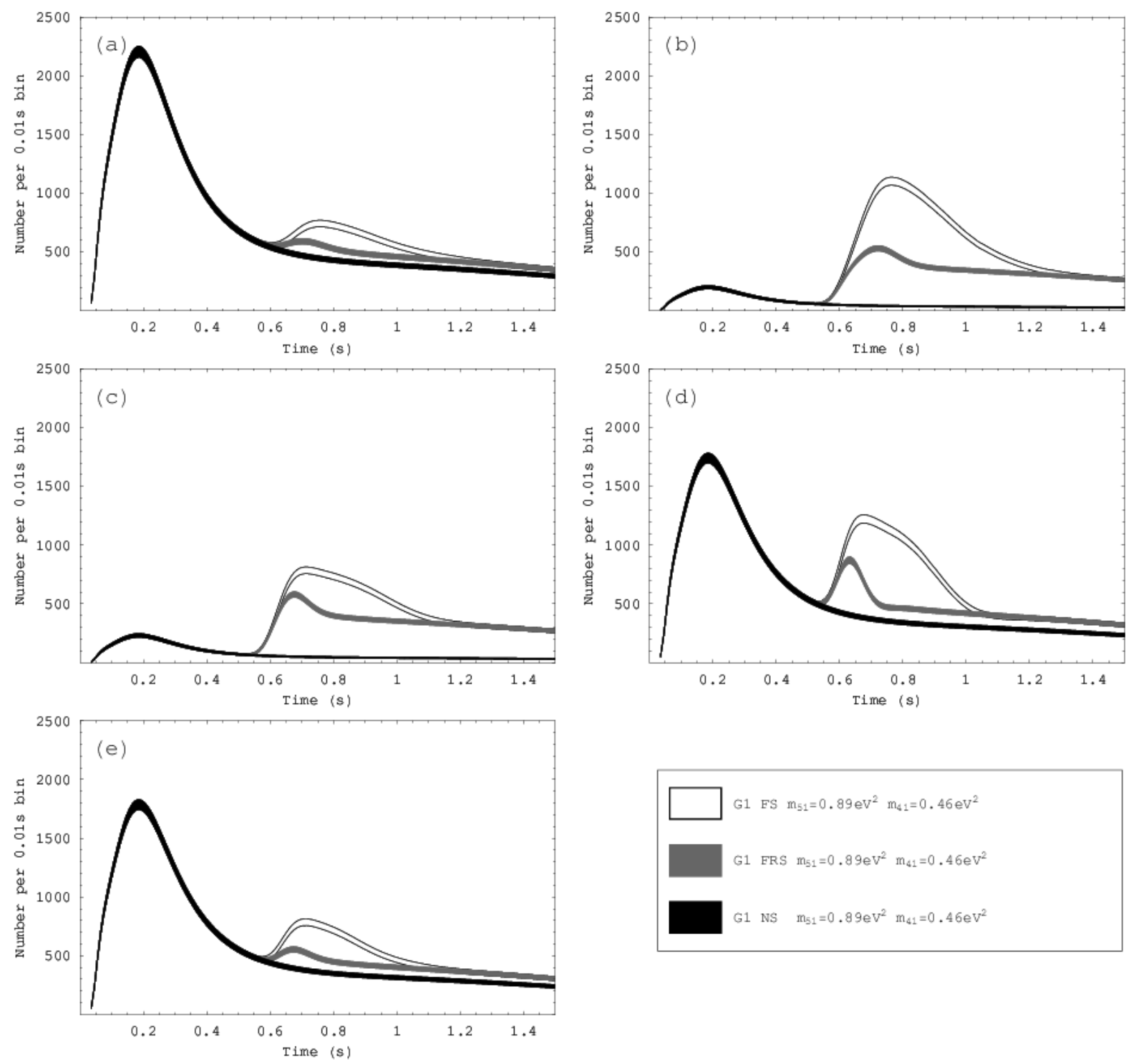

Figure 8: The number of events in $10 \mathrm{~ms}$ bins, for the cases of $\Delta m_{41}^{2}=0.46 \mathrm{MeV}^{2}$ and $\Delta m_{51}^{2}=0.54 \mathrm{MeV}^{2}$, with turbulence, a forward shock (FS), a forward and reverse shock (FRS) and no shock (NS), with the initial energy spectra G1 as discussed in the text:(a)N2 + N3, (b)N2 + I3, (c)H2 + N3, (d)H2 + I3, (e)I2 + N3. 

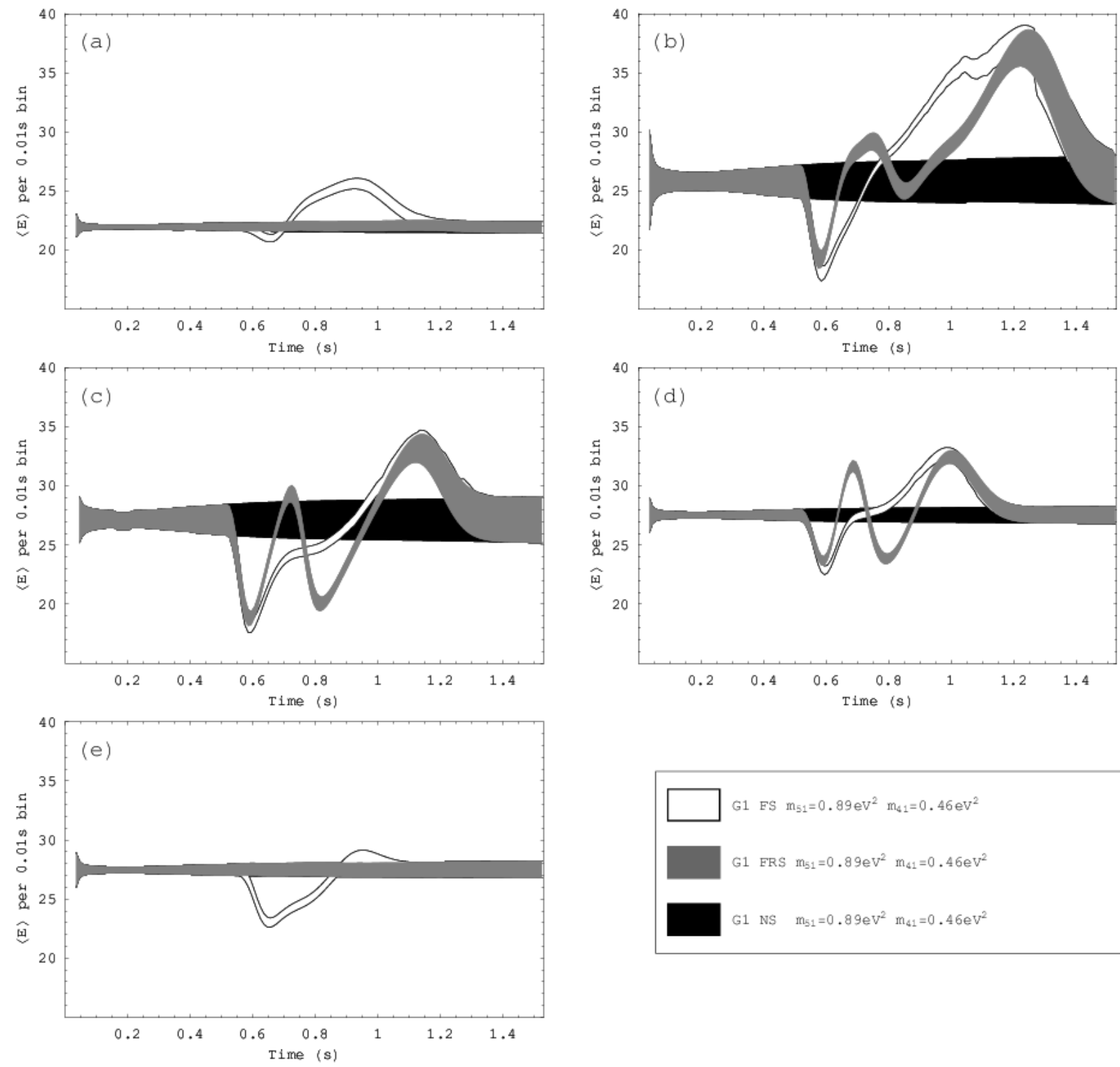

Figure 9: The average energy in $10 \mathrm{~ms}$ bins, for the cases of $\Delta m_{41}^{2}=0.46 \mathrm{MeV}^{2}$ and $\Delta m_{51}^{2}=0.54 \mathrm{MeV}^{2}$, with no turbulence, a forward shock (FS), a forward and reverse shock (FRS) and no shock (NS), with the initial energy spectra G1 as discussed in the text:(a)N2 + N3, (b)N2 + I3, (c)H2 + N3, (d)H2 + I3, (e)I2 + N3. 

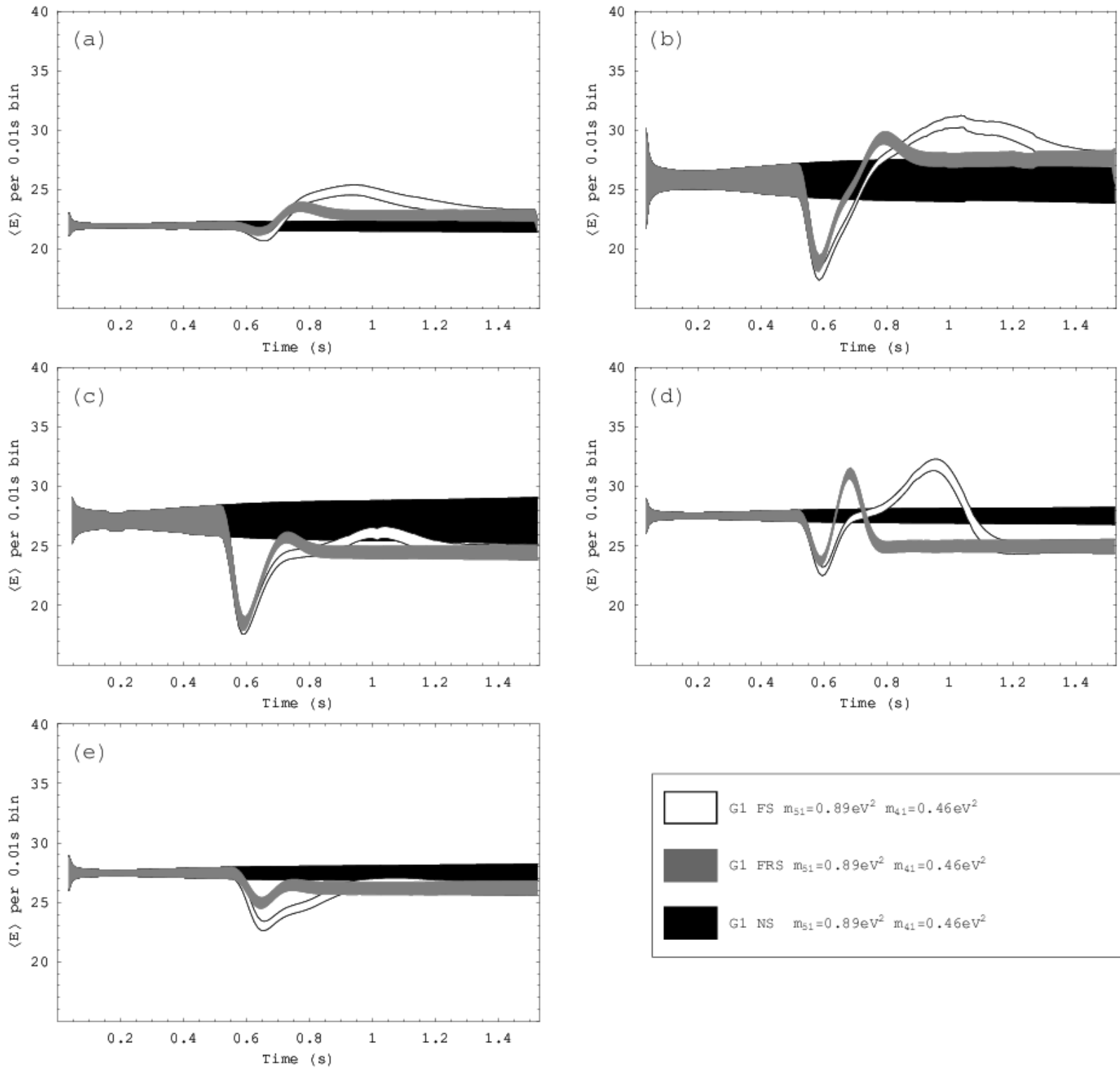

Figure 10: The average energy in $10 \mathrm{~ms}$ bins, for the cases of $\Delta m_{41}^{2}=0.46 \mathrm{MeV}^{2}$ and $\Delta m_{51}^{2}=0.54 \mathrm{MeV}^{2}$, with turbulence, a forward shock (FS), a forward and reverse shock (FRS) and no shock (NS), with the initial energy spectra G1 as discussed in the text:(a)N2 + N3, (b)N2 + I3, (c)H2 + N3, (d)H2 + I3, (e)I2 + N3. 

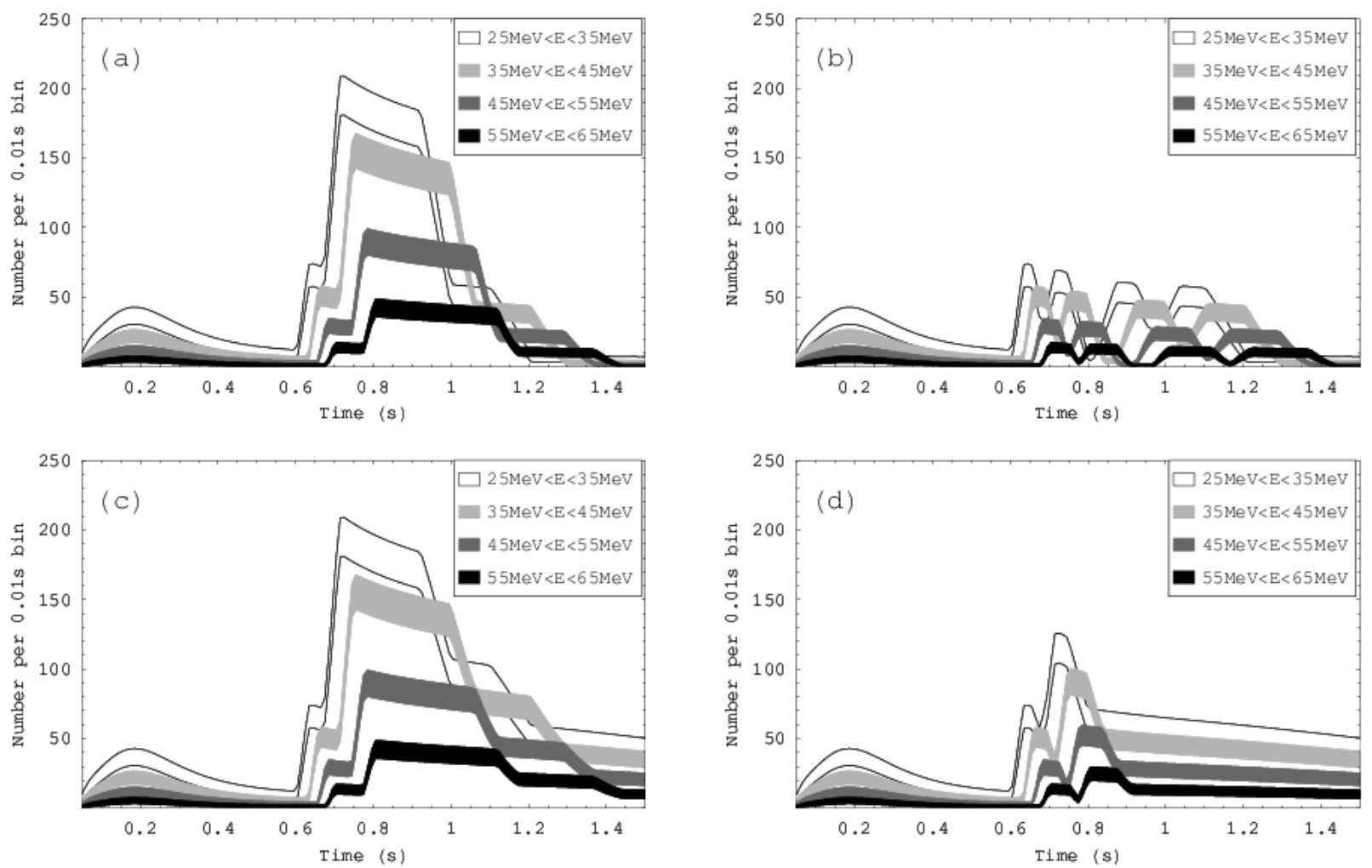

Figure 11: The number of events in $10 \mathrm{~ms}$ and $10 \mathrm{MeV}$ bins, for $\mathrm{N} 2+\mathrm{I} 3$ and the cases of a forward shock (FS), a forward and reverse shock (FRS), with the initial energy spectra LL as discussed in the text:(a)FS no turbulence, (b)FRS no turbulence, (c)FS with turbulence, (d)FRS with turbulence. 